\title{
Hysteretic Behavior of Moment-Resisting Frames Considering Slab Restraint and Framing Action
}

Abstract: This paper examines the influence of the framing action and slab continuity on the hysteretic behavior of composite-steel moment-resisting frames (MRFs) by means of high-fidelity continuum finite element (CFE) analyses of two-bay sub-systems and typical cruciform subassemblies. The CFE model, which is made publicly available, is thoroughly validated with available full-scale experiments and considers variations in the beam depth and the imposed

11 loading history. The simulation results suggest that beams in sub-systems may experience up to

$1225 \%$ less flexural strength degradation than those in typical subassemblies. This is due to local

13 buckling straightening from the slab continuity and framing action evident in sub-systems. For the

14 same reason, beam axial shortening due to local buckling progression is up to five times lower in 15 sub-systems than in subassemblies, which is consistent with field observations. While the hysteretic 16 behavior of interior panel zone joints is symmetric, exterior joint panel zones in sub-systems 17 experience large asymmetric shear distortions regardless of the employed lateral loading history.

18 From a design standpoint, it is found that the probable maximum moment in deep and slender 19 beams ( $d_{b} \geq 700 \mathrm{~mm}$ ) may be up to $25 \%$ higher than that predicted by current design provisions 20 with direct implications to capacity design of steel MRFs. The $25 \%$ reduction in the shear stud 21 capacity as proposed by current seismic provisions is not imperative for MRFs comprising 22 intermediate to shallow beams and/or featuring a high degree of composite action $(\eta>80 \%)$ as 23 long as ductile shear connectors are employed.

24 Keywords: Composite-steel moment resisting frames; Slab restraint; Frame continuity; 25 Continuum finite element analysis; Cyclic deterioration; Panel zone shear distortion; Lateral load 26 protocol; Shear stud behavior

\footnotetext{
${ }^{1}$ PhD candidate, RESSLab, ENAC, École Polytechnique Fédérale de Lausanne (EPFL), Switzerland. E-mail: hammad.eljisr@epfl.ch

${ }^{2}$ Lecturer, University of Southampton, Southampton, United Kingdom. E-mail: a.elkady@,soton.ac.uk (formerly, Postdoctoral research scientist, RESSLab, ENAC, École Polytechnique Fédérale de Lausanne (EPFL), Switzerland)

3* (Corresponding Author) Associate professor, RESSLab, ENAC, École Polytechnique Fédérale de Lausanne (EPFL), Switzerland. E-mail: dimitrios.lignos@epfl.ch
} 


\section{Introduction}

The 1994 Northridge and 1995 Kobe earthquakes led to a paradigm shift in the seismic design of steel moment resisting frames (MRFs). As part of the SAC ${ }^{1}$ project (Mahin 1998), multiple testing programs were conducted on beam-to-column subassemblies (Engelhardt et al. 2000; FEMA 2000b; Ricles et al. 2002; Sumner and Murray 2002; Tremblay et al. 1997; Uang et al. 2000; Zhang et al. 2004). These tests formed the basis for the development of today's pre-qualified beam-to-column connections for seismic applications in the US (AISC 2016a). A concerted effort is currently underway in Europe (Landolfo et al. 2018) regarding the same matter.

The significant majority of the tests conducted as part of the SAC project involved subassemblies with $\mathrm{T}$ - or cruciform-shaped configurations. While these subassemblies may be convenient for physical testing due to their overly-simplified boundary conditions, they do not represent reality at damage states associated with large inelastic deformations (Cordova and Deierlein 2005; Zerbe and Durrani 1989). In particular, beams in cruciform subassemblies are free to shorten axially (Civjan et al. 2001; MacRae et al. 2013) after the formation of local buckling within the anticipated dissipative zone of a steel beam. This is not evident in system-level tests (Cordova and Deierlein 2005; Del Carpio et al. 2014) and field observations (Clifton et al. 2011; Okazaki et al. 2013), where beam local buckling is delayed due to the axial restraint provided by the slab continuity (Cordova and Deierlein 2005; Donahue et al. 2017; FEMA 2000a; Herrera et al. 2008). Moreover, the floor slab and adjacent columns in buildings provide restraint to the beam and inhibit axial shortening (PEER/ATC 2010). This may result in an appreciable increase in the plastic rotation capacity of the steel beam (FEMA 2000a; Kwasniewski et al. 2002). Although inconclusive, El Jisr et al. (2019) highlighted that the plastic rotation capacity of composite steel beams directly deduced from system-level or sub-system tests may be at least two times larger than that deduced from beams in cruciform configurations.

The effect of the slab axial restraint may have significant implications on nonlinear modeling of steel MRF beams. Common numerical modeling approaches include point plastic hinge models as well as distributed finite-element approaches (Deierlein et al. 2010). While point hinge (Elkady

\footnotetext{
${ }^{1} \mathrm{SAC}$ is a joint venture of the Structural Engineers Association of California (SEAOC), the Applied Technology Council (ATC) and California Universities for Research in Earthquake Engineering (CUREe)
} 
and Lignos 2014; Ibarra et al. 2005; Lignos and Krawinkler 2011; Rassati et al. 2004) and resultant section models (El-Tawil and Deierlein 2001; Mehanny and Deierlein 2000) for composite steel beams are available in the literature, they have been established on the basis of subassembly tests. Hence, potential differences, due to the framing action, in the hysteretic behavior of beams between interior and exterior joints of a steel MRF are ignored. Furthermore, these models typically neglect the interface slip between the steel beam and the concrete slab. These effects may be captured with fiber models (Amadio and Fragiacomo 1993; Ayoub 2005; Ayoub and Filippou 2000; Bursi et al. 2005; Bursi and Ballerini 1996; Gattesco 1999; Salari and Spacone 2001). Albeit these models are computationally efficient, they require effective stress-strain formulations with softening to trace strength and stiffness deterioration (Kolwankar et al. 2018; Suzuki and Lignos 2018). In the absence of comprehensive experimental data, continuum finite element (CFE) models offer a rational alternative to quantify the aforementioned effects. Past studies involving CFE models (Alashker et al. 2010; Elkady and Lignos 2015b, 2018a; Zhang et al. 2004; Zhou et al. 2007) focused mostly on the dependence of strength and stiffness deterioration of steel members on nonlinear geometric instabilities (e.g., local and/or lateral torsional buckling). To the best of the authors' knowledge, there are no comprehensive studies to elucidate the physical mechanisms associated with the slab axial restraint at interior and exterior beam-to-column joints within a subsystem (entire story) and/or structural system.

From a seismic design standpoint, headed shear studs transfer seismic inertia forces through the slab into the MRF steel beams. Early degradation in the shear stud strength results in the loss of this load-transfer mechanism, thereby triggering loss of composite action (Cheng and Chen 2005; Civjan et al. 2001; Leon et al. 1998). As a precaution against severe shear strength degradation of the studs, current seismic provisions (AISC 2016b; CEN 2004a) propose a 25\% reduction in the stud design shear resistance. Albeit this reduction may be rational in steel MRFs comprising deep beams (depths larger than $400 \mathrm{~mm}$ ), it is not justifiable in prospective steel MRF designs comprising shallow composite beams with a high degree of composite action, $\eta \geq 80 \%$ ( $\eta$ is the ratio of the actual number of shear studs to that required to achieve full composite action). This perception has been mostly put in place based on cyclic push-out tests (Bursi and Gramola 1999; Civjan and Singh 2003; Zandonini and Bursi 2002). Although informative, these tests do not replicate the actual stress state and boundary conditions in the slab due to bending, nor do they 
account for the force redistribution between the studs (Schafer et al. 2019; Sjaarda et al. 2018; Suzuki and Kimura 2019).

This paper addresses all the aforementioned issues by means of CFE analyses. The proposed CFE modeling approach, which is validated on the basis of composite subassembly tests, explicitly accounts for the synergy between the composite slab and the steel beams. The modeling approach is extended to two-bay sub-systems to comprehend the influence of the slab axial restraint and framing action on the hysteretic behavior of beams and panel zones in interior/exterior beam-tocolumn joints. These sub-systems comprise beams with depths representative of both the North American and European seismic design practice. Aspects associated with the shear stud resistance in contemporary designs of composite steel MRFs are discussed.

\section{Proposed Continuum Finite Element Modeling Approach}

This section discusses the CFE modeling specifics of a typical beam-to-column subassembly with a composite floor slab as illustrated in Fig. 1(a). The modeling approach is validated with data from a full-scale subassembly test (Zhang et al. 2004) featuring fully-restrained beam-tocolumn connections with reduced beam sections (RBS). The commercial finite element software Abaqus 6.14 (Abaqus 2014) is used for this purpose. Referring to Fig. 1, column and beam regions within contact zones are meshed with first-order brick elements with incompatible modes, $C 3 D 8 I$. These elements are suitable for nonlinear analysis involving contact (Selamet and Garlock 2010). They are fully integrated with additional internal degrees of freedom to eliminate shear locking and they capture bending with an accuracy similar to that of quadratic elements. A structured mesh is implemented in the beam's anticipated plastic hinge location to produce elements with reasonable aspect ratios. Three elements per flange thickness are considered as recommended by Bursi and Jaspart (1998) for flexure-dominated problems. The remaining beam and column regions are modeled using four-node double-curved $S 4 R$ shell elements with five integration points along the element thickness based on the recommendations by (Elkady and Lignos 2018a). A shell-tosolid coupling constraint is used to connect the shell edge regions of the beam to the column flange. The concrete slab is modeled using eight-node first-order brick elements with reduced integration, $C 3 D 8 R$. Five elements across the slab thickness are considered to provide satisfactory performance against hourglassing (Genikomsou and Polak 2015). Slab rebar and wire mesh reinforcement are 
113

114

115

116

117

118

119

120

121

122

123

124

125

126

127

128

129

130

131

132

133

134

135

136

137

138

139

140

141

142

143

modeled using two-node linear truss elements, T3D2. The steel deck is modeled using four-node membrane elements, $M 3 D 4 R$.

Shear studs connecting the slab to the transverse beams are modeled with two-node linear beam elements, $B 31$. The cross-sectional area of these elements is modified to make it equivalent to the actual shear stud strength and stiffness (Baskar et al. 2002; Liang et al. 2005). The shear studs between the main beams and the slab may exhibit a pinched hysteretic degrading response. To capture this response, the interface slip should be modeled using a nonlinear load-slip behavior (Ayoub and Filippou 2000; Bursi et al. 2005). For this purpose, a user-defined element (VUEL) is developed by the authors and implemented as shown in Fig. 1(d). This VUEL, which is publicly available from https://github.com/eljisr/IMK_Pinching_VUEL, employs the modified IbarraMedina-Krawinkler (IMK) deterioration model (Ibarra et al. 2005; Lignos and Krawinkler 2011). The model assumes a pinched hysteretic behavior that explicitly simulates the effects of stiffness, strength, post-capping strength and accelerated reloading stiffness deterioration (Ibarra et al. 2005). It is loading-history independent and assumes a reference inherent hysteretic energy dissipation capacity regardless of the applied protocol. The set of parameters that define the deterioration model are calibrated with cyclic push-out tests available in literature. Past conventional cyclic push-out tests have demonstrated severe degradation in the shear studs (Bursi and Gramola 1999; Civjan and Singh 2003; Zandonini and Bursi 2002). However, they do not accurately replicate the mechanical behavior of the shear stud connectors under fully reversed cyclic loading. More importantly, they do not consider the force redistribution occurring in the studs once they experience cyclic deterioration. For this reason, the shear studs are calibrated with recently conducted cyclic-push out tests that account for the stress state in the slab under reversed cyclic loading (Suzuki and Kimura 2019). These tests were subjected to symmetric loading protocols, which impose far higher inelastic demands to studs than non-symmetric loading protocols. In that respect, the calibration is on the conservative side. Figure 2 shows a calibration of the hysteretic behavior of a cluster of four $19 \mathrm{~mm}$ shear studs. Based on this calibration, the following parameters are obtained for a single stud (positive and negative superscripts refer to the stud parameters when the slab is under compression and tension, respectively): the ultimate shear strengths $Q_{u}^{+}=82 \mathrm{kN}$ and $Q_{u}^{-}=36 \mathrm{kN}$, the effective yield strengths $Q_{y}=90 \%$, the pre-capping slip capacities $s_{p}^{+}=$ $6 \mathrm{~mm}$ and $s_{p}^{-}=10 \mathrm{~mm}$, the post-capping slip capacities $s_{p c}^{+}=11 \mathrm{~mm}$ and $s_{p c}^{-}=5 \mathrm{~mm}$, the ultimate slip capacities $s_{u}^{ \pm}=15 \mathrm{~mm}$, the strength and stiffness deterioration parameters $\lambda_{s}=40$ and 
$144 \lambda_{k}=15$, the deterioration rate parameters $D^{ \pm}=1.0$, and the parameters that define the break point 145 of the pinching model $\kappa_{d}=0.4$ and $\kappa_{f}=0.2$.

146 The steel material multi-axial constitutive relationship for beams and columns is based on the 147 well-established Voce-Chaboche multiaxial plasticity model (Lemaitre and Chaboche 1994; Voce 148 1948). The input model parameters are adopted based on studies by Sousa and Lignos (2018) for 149 A992 Gr. 50 steel (ASTM 2015). An elastic perfectly-plastic material model is assigned to the 150 steel deck and slab reinforcement.

151 The concrete behavior under cyclic loading is simulated through the concrete damaged 152 plasticity (CDP) model, available in Abaqus 6.14 (Abaqus 2014). The yield function of this model 153 under multiaxial stress state accounts for damage in the concrete (Lee and Fenves 1998; Lubliner 154 et al. 1989). The plastic flow potential is defined using the Drucker-Prager hyperbolic function. 155 The parameters recommended by Goto et al. (2010) are implemented in the developed CFE model. 156 The stress-strain relation of concrete under compression is defined according to Carreira and Chu 157 (1985) and Baskar et al. (2002). The concrete compressive strength is $f_{c}^{\prime}=32 \mathrm{MPa}$. Under tension, 158 a linear elastic behavior is assumed up to the concrete tensile strength, $f_{t}=10 \% f_{c}^{\prime}$ (Matsumura 159 and Mizuno 2007). A tension stiffening strain of 0.1 is employed (Baskar et al. 2002; Rex and 160 Easterling 2000). Stiffness degradation mechanisms are incorporated in the CDP model through 161 compressive and tensile damage variables (Goto et al. 2010).

162 Referring to Fig. 1(a), the pinned boundary conditions assumed at the main beams and column 163 ends correspond to those expected at the inflection point locations in a typical MRF under lateral 164 loading. Out-of-plane movement and twisting are restrained at the main beams and column ends 165 at the indicated points shown in Fig. 1(a). The transverse floor beams supporting the floor slab are 166 connected to the main beams via a conventional shear tab connection (see Fig. 1(c)). A tie 167 constraint is used to idealize this connection for the translational degrees of freedom. Therefore, 168 the connection can resist moment under strong-axis bending only. This assumption is based on the 169 fact that the shear-tab connection is not an ideal pin and has a non-negligible strong-axis rotational 170 strength and stiffness (Liu and Astaneh-As1 2004). Furthermore, the strong-axis flexural demand 171 on the connection (due to twisting) is low and is mainly resisted by the diagonal brace and the slab.

172 On the other hand, under weak axis bending, the rotational stiffness and flexural strength of the 173 shear tab connection is negligible. The out-of-plane movement of the main beam's bottom flanges 
is prevented with diagonal braces connected to the transverse beams. Modeling of these braces is simplified by employing a kinematic coupling constraint as shown Fig. 1(c).

The steel beams are rigidly connected to the column through a surface-based tie constraint. Continuity plates are fully tied to the column web and flanges, while doubler plates are tied to the column web at their edges. Plug welds are modeled using connector elements with an influence radius equal to that of the plug weld radius, and fully-constrained degrees of freedom (see Fig. 1(b)). The shear studs are connected to the beam and slab through multi-point beam constraints. A perfect bond is assumed between the concrete slab, and the rebar reinforcement. The inner surface of the steel deck is fully tied to the concrete slab.

Both the restraint provided by the slab to the top beam flanges, and the bearing of the slab on the column flanges are simulated using a general contact interaction. The interface action between the slab and the steel components consists of a hard contact relationship with balanced masterslave weighing and allowed separation. The friction behavior is expressed using a Coulomb model with a steel-to-concrete friction coefficient, $\mu=0.2$ (Johansson and Gylltoft 2002).

Local and global imperfections are introduced in the dissipative zones (i.e., RBS region) to properly trigger nonlinear geometric instabilities based on the modeling procedures proposed by (Elkady and Lignos 2018a). Residual stresses are also modeled using the distribution proposed by Young (1972).

\section{Validation of the Modeling Approach}

The proposed CFE modeling approach is validated with the subassembly specimen, SPEC3 from Zhang et al. (2004). The specimen features W36x150 main girders, a W27x194 column, W14x22 transverse beams and a $133 \mathrm{~mm}$ (5.25") slab. The floor slab is $1219 \mathrm{~mm}$ (4') wide, with a $305 \mathrm{~mm}$ (12") overhang. It consists of an $83 \mathrm{~mm}$ (3.25”) concrete fill (32 MPa) on top of a $51 \mathrm{~mm}$ (2") deep Vulcraft 2VLI steel deck, oriented such that the ribs are parallel to the main girders. The slab reinforcement includes a W4xW4 welded wire mesh, as well as No. $3(9.5 \mathrm{~mm})$ and No. 4 $(12.7 \mathrm{~mm})$ bars. Nine $19 \mathrm{~mm}(0.75$ ") shear studs connect each main girder to the slab. A single $12.7 \mathrm{~mm}(0.5$ ") doubler plate is welded to the column web. The specimen was subjected to a cyclic symmetric loading history (SAC Joint Venture 1997) at the column tip.

The nonlinear quasi-static analysis is run at EPFL's high performance computing center (Fidis Cluster) using a Message Passing Interface-based domain decomposition parallel implementation. 
The Abaqus/Explicit dynamic analysis procedure is employed. This procedure has a robust contact functionality to solve very complex contact problems (Prior 1994). This is critical for simulating the slab restraint to the top beam flange and the slab bearing on the column. In particular, the loading rate is assumed to be sufficiently small to ensure that the inertial force is nearly zero (i.e., equivalent to static loading). The main drawback of the explicit solution technique is that the time step is limited by the size of the stable time-increment, $t_{\text {stable }} \leq 2 / \omega_{\max }$, where $\omega_{\max }$ is the highest element eigenfrequency in the model. To overcome this shortcoming, the stable timeincrement is increased through mass-scaling. Quasi-static response is verified through the equilibrium of static forces and the energy balance in the model. Referring to Fig. 3, the ratio of the kinetic and viscous energies to the internal energy is less than $5 \%$ and the total energy in the model is nearly zero (Chung et al. 1998; Prior 1994). Artificial strain energy due to hourglassing control as well as distortion control dissipation energy are examined and found to be negligible.

Figures 4( $\mathrm{a}$ and $\mathrm{b}$ ) shows a comparison between the measured and simulated hysteretic response of the composite steel beam. In this figure, $M_{b}$ is the beam moment at the column face; $V_{P Z}$ is the panel zone shear force; $S D R_{b}, S D R_{P Z}$, and $S D R_{c}$ are the beam, panel zone and column contributions to the story drift ratio, respectively. Referring to Fig. 4(a), the CFE model predicts the onset of local buckling fairly well under sagging (slab in compression) and hogging (slab in tension) bending excursions. The predicted flexural strength and stiffness of the beam possesses an outstanding agreement with the measured one up to $6 \%$ story drift ratio. Deviation from the test results occurs in the last sagging excursion as ductile tearing initiated in the bottom flange of the

224 beam during the test. Figure 4(b) shows that the model marginally over-predicts the panel zone 225 deformations by about $10 \%$. This is due to the slightly higher predicted beam moment. However, 226 the panel zone contribution to the story drift, in both the CFE model and test, does not exceed 1\%. 227 Consequently, the slight deviation in the panel zone response does not practically influence the energy dissipation capacity of the beam-to-column connection as shown in Fig. 4(d).

Figure 4(c) demonstrates a noteworthy agreement between the predicted and measured 230 decomposed deformation contributions to the story drift. Referring to Fig. 4(d), the same 231 observations hold true with regards to the accumulated energy dissipated by each component. Note 232 that the peak deformation in the panel zone, in both the CFE and the test, occurs at 3\% SDR. At $6 \%$ SDR, the demand on the panel zone drops substantially as the beams experience flexural 
strength degradation. Accordingly, the panel zone contribution at 6\% SDR is negligible in both the CFE and the test ( $0.03 \%$ and $0.15 \%$ respectively).

\section{Parametric Study with Two-bay Sub-system Models}

Having established confidence in the CFE modeling approach, the effects of the floor slab continuity and framing action on the seismic performance of steel MRFs is assessed. The presence of neighboring gravity frames is also expected to provide some degree of additional restraint on the steel MRF (Donahue et al. 2017), thereby enhancing these effects. However, the influence of gravity framing is not considered in this paper. Three two-bay sub-systems, summarized in Table 1 , are considered herein: $S_{D}$ with deep beams, $S_{I}$ with beams of intermediate depth, and $S_{S}$ with shallow beams. The sub-systems cover a range of beam sizes employed in typical low to mid-rise steel MRF buildings (Elkady and Lignos 2014, 2015a; Tartaglia et al. 2018; Tsitos et al. 2018). The centerline span length, $L=8992 \mathrm{~mm}$ (29-1/2'), column height, $H=3962 \mathrm{~mm}$ (13'), maximum unbraced length, $L_{b}=1676 \mathrm{~mm}$ (5-1/2'"), and the slab dimensions correspond to those of subassembly specimen SPEC3 (Zhang et al. 2004). In all three sub-systems, the member slenderness ratio $L_{b} / r_{y}$, summarized in Table 1, is compliant with ANSI/AISC 341-16 (AISC 2016b) for special moment frames. The shear span-to-depth ratio $L_{o} / d_{b}$ of specimen $\mathrm{S}_{\mathrm{D}}$ does not quite satisfy the ductility requirements of ANSI/AISC 341-16 (AISC 2016b). Nonetheless, the moment-shear interaction was found to be insignificant, especially in the absence of gravity load. This is consistent with available test data on composite connections (El Jisr et al. 2019). Since the maximum shear force that can be transferred through the shear studs is governed by the capacity of the concrete slab, the degree of composite action, as defined by ANSI/AISC 360-16 (AISC $2016 \mathrm{c})$, is the same for all three sub-systems $(\eta \sim 20 \%)$. The columns are sized to remain elastic (see strong-column-weak-beam (SCWB) ratio in Table 1), whereas the web panel zones are sized to comply with ANSI/AISC 341-16 (AISC 2016b). Equal displacement was imposed at the top of the columns. This loading technique assumes a rigid diaphragm for the floor slab above the considered sub-system. The sub-systems are subjected to a cyclic symmetric lateral loading history up to an SDR of 6\% (SAC Joint Venture 1997). Sub-system $S_{D}$ is also subjected to a collapseconsistent protocol (Suzuki and Lignos 2019) to investigate the influence of loading history on the sub-system cyclic performance. For each sub-system, the seismic behavior is compared with that of the corresponding interior joint subassembly featuring simplified boundary conditions. 
Particular emphasis is placed on the hysteretic behavior of the composite beams, panel zones, and shear studs, the accumulated beam axial shortening and beam axial force demands.

\section{Lateral Drift Demand Contributions}

The deformation demands at the interior and exterior joints of the sub-systems are examined. Figure 5 depicts the decomposed deflection contributions to the story drift ratios of specimens $S_{D}$ and $S_{S}$ at selected SDRs. In particular, the columns remain elastic, as intended, with minimal contribution to the SDR. At the interior joint of $S_{D}$ and at $4 \%$ lateral drift demand (Figs. 5(a and b)), the panel zone contribution to the story drift is around $35 \%$. This is considerably higher than the panel zone contribution in the corresponding interior joint subassembly (see Fig. 4(c)). The axial restraint in the sub-system delays the flexural strength degradation in the beams, thereby increasing the panel zone shear demand. At 6\% drift amplitude, when beam local buckling becomes more evident and the inelastic deformations concentrate in the beam, the panel zone contribution to the story drift decreases to about $10 \%$. While a similar behavior is observed in $\mathrm{S}_{\mathrm{S}}$ (Figs. 5(c and d)), the panel zone contribution to the story drift remains appreciable $(\sim 25 \%)$ at $6 \%$ drift amplitude. Flexural strength degradation in shallow beams is minimal as discussed in the following section. Notably, exterior joints exhibit a distinct asymmetric behavior. Therefore, the panel zone contribution to the story drift is dependent on the direction of lateral loading. Particularly, the demand on the panel zone is higher when the framing beam is subjected to sagging (Figs. 5(a and c)) compared to hogging bending (Figs. 5(b and d)). The reasons behind this asymmetric demand are investigated more thoroughly in the subsequent sections. Referring to Fig. 5 (c), the exterior joint panel zone contribution to the $6 \%$ story drift is nearly $40 \%$ despite being designed with a resistance-to-demand ratio, $R_{v} / V_{d}=1.9\left(R_{v}\right.$ and $V_{d}$ are defined in Table 1$)$. Moreover, at large lateral drift demands ( $S D R \geq 4 \%$ ), the exterior joint panel zones deform in one loading direction although the lateral drift demand is symmetric as shown in Figs. 5(b and d). In order to offset this negative contribution of the panel zone, the beam contribution to the story drift at the exterior joints exceeds the imposed drift demand. The behavior is nearly identical at both exterior joints of the sub-systems.

\section{Beam Hysteretic Response}


Referring to Fig. 6, the hysteretic response of the west beam is obtained for all three configurations and compared to that of the corresponding interior joint subassembly. For reference,

$294 M_{b, W e s t, C 1}$ and $M_{b, W e s t, C 2}$ are the west beam moments at the face of columns C1, and C2, 295 respectively. Referring to Fig. 6(a), the beam flexural strength degradation under sagging bending 296 occurs at a fairly slow rate, even at 6\% lateral drift demand, in the interior joint of sub-system $297 S_{D}$ when compared to that of the corresponding subassembly. Table 2 shows that beams in the two298 bay sub-systems may experience up to $25 \%$ less flexural strength degradation, than those in 299 subassemblies under symmetric-cyclic lateral loading. This particularly applies to deep and slender 300 beams that are prone to local buckling (Lignos and Krawinkler 2011). This behavior is attributed 301 to the restraint provided by the floor slab and adjacent columns against the beam axial shortening, 302 which results in the straightening of the beam local buckles. The straightening effect is more 303 evident under hogging than sagging bending. Figure 6(a) shows that under hogging bending, the 304 strength degradation is only slightly lower in the sub-system than in the subassembly. The beam 305 hysteretic response at the interior joint of sub-systems $S_{\mathrm{I}}$ and $S_{\mathrm{S}}$ shows minimal flexural strength 306 degradation, similar to that of the corresponding subassemblies. The former has a low web 307 slenderness ratio, $h_{b} / t_{w}=31.3$, which delays the formation of web and flange local buckling at 308 large inelastic cycles (Lignos and Krawinkler 2011). On the other hand, sub-system $\mathrm{S}_{\mathrm{S}}$ consists 309 of a shallow steel beam; as such, the slab contribution to the flexural resistance of the composite 310 beam is higher than that in $S_{D}$ and $S_{I}$. This results in a lower compressive stress in the top beam 311 flange, thereby limiting local buckling under sagging bending.

312 Beam flexural strength degradation under hogging bending occurs as a result of the formation 313 of large buckles in the bottom flange of the beam. In a sub-system, these buckles are straightened 314 out during the sagging bending excursions due to the slab restraint against axial shortening. This 315 agrees with earlier observations from physical testing of composite-steel MRFs (Cordova and 316 Deierlein 2005). Furthermore, the beam and slab continuity at the interior joint augments this 317 restraint (Cordova and Deierlein 2005; Herrera et al. 2008). Referring to Figs. 7(a and b), the 318 buckled portions of the beam web and flanges experience notable straightening upon load reversal. 319 As a result, pinching behavior, caused by an increase in the rotational stiffness of the composite 320 beams, is observed in their hysteretic response (see Fig. 6). The axial restraint induces additional 321 tensile axial forces $\left(F_{a, W}^{ \pm}\right.$and $\left.F_{a, E}^{ \pm}\right)$and moments $\left(M_{a, W}^{ \pm}\right.$and $\left.M_{a, E}^{ \pm}\right)$in the beams. The latter are 322 caused by non-uniform buckling along the beam depth. On the other hand, beams in cruciform 
subassemblies are free to shorten at their ends due to the simplified boundary conditions, resulting in an "accordion" effect due to the build-up of local buckles. Figures 7(c and d) suggest that, in a subassembly, beam flanges that buckled experience minor straightening upon load reversal. A comparison between Figs. 7(a and b) and Figs. 7(c and d) reveals that the extent of bottom flange local buckling is closely akin in subassemblies and sub-systems. Hence, the rate of strength degradation under hogging bending is also expected to be cognate. This is not the case for sagging bending. First, the net tensile axial force acting on the beam is larger under sagging than hogging bending $\left(\left|F_{a, E / W}^{+}+F_{b, E / W}^{+}\right|>\left|F_{a, E / W}^{-}+F_{b, E / W}^{-}\right|\right)$. Second, the additional moment induced by nonuniform buckling along the beam depth is lower under sagging than hogging bending $\left(\left|M_{a, E / W}^{+}\right|<\right.$ $\left.\left|M_{a, E / W}^{-}\right|\right)$due to the restraint provided by the slab to the top flange of the beam. Third, the rate of stud degradation is lower in sub-systems when compared to that of subassemblies. The composite action is maintained even at large lateral drift demands (SDR $\geq 4 \%$ ), which alleviates the compressive force near the top flange and enhances it near the bottom flange.

Referring to Figs. 6(b, d and f), the beam hysteretic response at the exterior joint of sub-systems is fully asymmetric despite the fact that the imposed loading history is symmetric. Particularly, the exterior joint beams experience flexural strength deterioration only under hogging bending. This behavior is a consequence of the asymmetric demand on the exterior column web panel zone. The mechanistic reason behind the observation above is explained in the next section.

Another consequence of the beam local buckling extenuation due to the slab restraint, is the underestimation of the probable maximum moment in the beam, $M_{f}$, calculated as per ANSI/AISC 358-16 (AISC 2016a). Figure 6 suggests that although $M_{f}$ is predicted fairly well for the subassembly featuring deep beams, it is underestimated by about $25 \%$ in sub-system $S_{D}$. The delay in local buckling in the beams results in additional cyclic hardening that does not occur in the subassembly. Additionally, since the North American design practice typically employs deep beams with a low degree of composite action, the slab contribution to $M_{f}$ is ignored according to ANSI/AISC 358-16 (AISC 2016a). Hence, the underestimation of $M_{f}$ is larger in sub-systems with shallower beams $\left(\mathrm{S}_{\mathrm{I}}\right.$ and $\left.\mathrm{S}_{\mathrm{S}}\right)$, where composite action is more pronounced. This issue is critical (a) for sizing columns to remain elastic based on the SCWB ratio; and (b) for estimating the panel zone shear demands. The implications of the latter are discussed in the next section. 


\section{Panel Zone Hysteretic Response}

The hysteretic response of the beam-to-column web panel zones is shown in Fig. 8 for both the interior and exterior joints. Referring to Figs. 8(a, c and e), the interior joint panel zones of the sub-systems experience more shear yielding than their subassembly counterparts. This is particularly true for specimens $S_{D}$ and $S_{S}$ in which higher moments are attained in the beams framing the joint. At $4 \%$ SDR, the shear distortion reaches $6 \gamma_{y}, 3.6 \gamma_{y}$ and $5.7 \gamma_{y}$ for specimens $\mathrm{S}_{\mathrm{D}}$, $\mathrm{S}_{\mathrm{I}}$ and $\mathrm{S}_{\mathrm{S}}$, respectively $\left(\gamma_{y}\right.$ is the shear distortion at initial yielding as defined according to ANSI/AISC 341-16 (AISC 2016b)). At exterior joints, the column web panel zone hysteresis shows a distinct asymmetric response (see Figs. 8(b, d and f)). The shear distortion in the exterior joint panel zones at $4 \%$ SDR reaches $7.3 \gamma_{y}, 8.5 \gamma_{y}$ and $5.2 \gamma_{y}$ for specimens $S_{D}, S_{I}$ and $S_{S}$, respectively. Despite being designed for a maximum distortion of $4 \gamma_{y}$ as per ANSI/AISC 341-16 (AISC 2016b), the composite action and axial restraint provided by the floor slab cause additional inelastic shear distortion. This is not expected to cause premature fracture in view of recent experimental findings (Shin and Engelhardt 2013). Interestingly, El Jisr et al. (2019) found that in composite beam-to-column connections, panel zones can develop a total shear distortion of $10 \gamma_{y}$ without experiencing premature fracture within the beam-to-column connection at a $5 \%$ lateral drift demand. However, for tall buildings, the excessive distortion in the panel zones may become a concern when considering second-order effects. The panel zone shear resistance at a given inelastic shear distortion should be compared with the respective shear demand from the intersecting beams and columns to avoid the formation of soft story mechanisms that could increase the collapse risk due to P-delta effects.

The asymmetry observed in the hysteretic response of the exterior column web panel zones is explained through the development of three mechanisms. The first two are related to the asymmetric flexural demand in the beam framing the exterior joint. That is, the flexural demand in the beam is higher under sagging than under hogging bending.

Mechanism 1 is a direct consequence of the composite action in the beam. The sagging flexural resistance of the beam is enhanced (up to $80 \%$ ) due to the composite action, while the hogging flexural resistance is also enhanced, but to a lesser degree (up to 40\%) depending on the slab reinforcement (El Jisr et al. 2019). The factor $\alpha_{1}$, shown in Fig. 9, accounts for this enhancement: 
$\alpha_{1}^{ \pm}=W_{p l, c}^{ \pm} / W_{p l}$ and $\alpha_{1}^{+}>\alpha_{1}^{-}>1.0\left(W_{p l, c}^{ \pm}\right.$is the plastic modulus of the composite section under 382 sagging $\left(W_{p l, c}^{+}\right)$or hogging $\left(W_{p l, c}^{-}\right)$bending, and $W_{p l}$ is the plastic modulus of the bare steel crosssection with respect to its strong axis). Furthermore, the presence of the slab increases the depth of the region in the steel cross-section subjected to compressive stresses under hogging bending and decreases it under sagging bending. Hence, flexural strength degradation is hastened under hogging excursions, and delayed under sagging excursions. The factor $\beta_{1}^{ \pm}$shown in Fig. 9, accounts for the phenomenon associated with the delay of local buckling under sagging bending $\left(\beta_{1}^{+}\right)$, and the progression of local buckling under hogging bending $\left(\beta_{1}^{-}\right)$in the composite beam: $\beta_{1}^{+}>1.0>\beta_{1}^{-}>0$. Mechanism 1 is more prominent in shallow beams $\left(d_{b} \leq 500 \mathrm{~mm}\right)$ where the effects of composite action are more pronounced compared to deep beams ( $\left.d_{b} \geq 700 \mathrm{~mm}\right)$.

Mechanism 2 involves the restraint that the slab provides to the top flange of the beam. The slab restraint delays the formation of local buckles, and hence the flexural strength degradation of the beam under sagging loading excursions. The flexural strength of the beam increases due to strainhardening. The factor $\alpha_{2}$, shown in Fig. 9(a), accounts for the additional strain hardening in the beam due to the restraint provided by the slab on the top beam flange: $\alpha_{2}>1.0$ regardless of the beam depth. The extent of the slab restraint to the top flange is dependent on the orientation of the steel deck. Cordova and Deierlein (2005) reported a higher restraint when the steel deck is oriented parallel to the beam. However, this issue is outside the scope of the present paper.

Mechanism 3 is caused by the axial restraint provided by the slab and the adjacent columns. This restraint induces a moment, as well as a net tensile force in the composite beam. The tensile force is non-uniform across the beam depth. That is, the axial force is comprised of a tensile force in the beam and a compressive force in the slab. Figures 9(a and b) show an idealization of the panel zone shear demand induced by the axial force in the composite beam. A force couple is assumed to act on the top and bottom locations of the panel zone. The factor $\gamma^{ \pm}>0$ represents the fraction of the composite beam axial force, $N_{b}$, acting in compression on the top beam flange under sagging $\left(\gamma^{+}\right)$and hogging $\left(\gamma^{-}\right)$bending respectively. Accordingly, the axial force increases the shear demand on the panel zone for sagging excursions by $\gamma^{+} N_{b}^{+}$(see Eq. (1)) and decreases it for hogging excursions by $\gamma^{-} N_{b}^{-}$(see Eq. (2)). Note that the $d_{e f f}^{ \pm}$defined in Fig. 9, is the effective depth of the panel zone as per the recommendations of Kim and Engelhardt (2002). Under hogging 
bending, $d_{e f f}^{-}$is equal to the distance between the centroid of the beam flanges, whereas under

411 sagging bending, $d_{e f f}^{+}$is equal to the distance between the centroid of the concrete section and 412 that of the beam bottom flange. Mechanism 3 is prevalent in beams that develop a large axial force 413 due to the axial restraint. Typically, these are deep beams $\left(d_{b} \geq 700 \mathrm{~mm}\right)$ with a low degree of 414 composite action $(\eta<50 \%)$ as will be explained in the following sections.

$V_{P Z}^{+}=M_{b, \max }^{+} / d_{e f f}^{+}-V_{T}^{+}+\gamma^{+} N_{b}^{+}$

$V_{P Z}^{-}=M_{b, \max }^{-} / d_{e f f}^{-}-V_{T}^{-}-\gamma^{-} N_{b}^{-}$

417 Mechanisms 1 and 2 appear to be the most dominant. This is based on findings from past 418 experiments on T-section subassemblies with composite floor slabs (Kim and Lee 2017; Yamada 419 et al. 2009). The tests showed a distinct ratcheting response in the web panel zones despite the 420 absence of axial restraint on the beam end. However, further studies should be conducted to 421 quantify the relative importance of each mechanism on the panel zone demand.

\section{Beam Axial Shortening}

The phenomenon of beam axial shortening has been observed in subassembly tests in which the steel beams are free to move axially at their ends (Civjan et al. 2001; FEMA 2000a; MacRae et al. 2013; Qi et al. 2018). Axial shortening occurs as local buckling builds up in the plastic hinge region (Cordova and Deierlein 2005). However, in an actual building, the axial restraint provided by the composite slab and adjacent columns is likely to limit this shortening. This is particularly true at interior joints where the slab is continuous, and for composite slabs with the deck ribs placed parallel to the steel girder (Civjan et al. 2001; Cordova and Deierlein 2005). Accordingly, the oversimplified subassembly boundary conditions may lead to overestimation of the extent of a beam's local buckling and subsequent axial shortening.

Figure 10a shows the definition of beam axial shortening, $\delta_{x}$, within a steel MRF bay. Referring to Fig. 10(b), subassembly $S_{D}$ beam experiences an excessive axial shortening of $50 \mathrm{~mm}$ at $6 \%$ 434 SDR. Top and bottom flange buckling mostly accumulate after 3\% SDR, which leads to a rapid progression of axial shortening. Figures 10 (c and d) shows that subassembly $S_{I}$ and $S_{S}$ beams do not shorten as much $(6 \mathrm{~mm}$ and $3 \mathrm{~mm}$ at $6 \%$ SDR respectively). The former comprises a $\mathrm{W} 21 \times 122$ beam with a fairly low $h_{b} / t_{w}=31.3$; the latter consists of a shallow beam $\left(d_{b}=409 \mathrm{~mm}\right)$ in 
which composite action is pronounced. In both specimens, the growth of local buckling across the beam depth is insignificant. Hence, axial shortening is minimal. Beams in sub-systems shorten much less compared to their subassembly counterparts. For instance, sub-system $S_{D}$ beam shortens by $7 \mathrm{~mm}$ at $6 \%$ SDR while sub-systems $S_{I}$ and $S_{S}$ beams do not practically experience shortening. The axial restraint provided by the composite slab and the adjacent columns alleviates the local buckling in the anticipated dissipative zone of the steel beam.

It is worth mentioning that beams in sub-systems $S_{D}$ and $S_{I}$ experience fairly minor elongation (up to 4mm). MacRae et al. (2013) attributed this elongation to the difference in the positions of the neutral axes at the beam ends. Under sagging bending, the neutral axis moves upwards towards the slab, whereas under hogging bending the neutral axis remains close to the beam centerline. This difference in the neutral axis positions is particularly noticeable in shallow beams. As a result, net centerline elongation results from tension yielding at the beam center near the sagging end, and compression yielding near the hogging end. Furthermore, the asymmetric shear distortion in the exterior joint panel zone exaggerates this net centerline elongation.

\section{Beam Axial Force}

Lateral loads are transferred to the column through the floor slab via two load paths (Cordova and Deierlein 2005; MacRae and Clifton 2015). The first one consists of direct bearing of the slab on the column face and a direct compression strut to the back of the column flange. The second load path involves the transfer of shear forces from the slab to the beam through friction and the shear studs. The resulting axial force in the beam is transferred to the column through the beamto-column connection. In subassemblies, the beam axial force at the location of the assumed inflection points is zero, increasing to its maximum value at the column face. In sub-systems, the axial restraint provided by the floor slab and columns causes an additional axial force in the beam. The magnitude of the beam axial force is not constant along the length of the beam and depends on the extent of the axial restraint. The beam axial force is higher near interior joints, where the axial restraint is higher, than near exterior joints. In sub-systems, unlike subassemblies, the axial force at the beam inflection point location is not zero. Accordingly, the additional axial force resulting from the axial restraint in sub-systems is quantified at the inflection point. 
Figure 11 shows the axial forces developed in the three sub-systems at the west beam inflection point. At 4\% SDR, the peak normalized tensile force ratio in the steel beam at the location of the inflection point $N_{s} / N_{p l}\left(N_{p l}=R_{y} F_{y b} A_{g}\right.$ as defined in Fig. 11(a)) is $8 \%, 2 \%$ and $5 \%$ for subsystems $S_{D}, S_{I}$ and $S_{S}$ respectively. The tensile force ratio at $6 \%$ SDR is $16 \%, 8 \%$ and $9 \%$ for subsystems $S_{D}, S_{I}$ and $S_{S}$ respectively. These values are expected to be higher near the interior joint. EN 1998-1 (CEN 2004a) states that the bending-axial force interaction in the steel beams may be disregarded as long as $N_{s} / N_{p l}<15 \%$. The floor slab is restrained by the shear studs, friction at the beam-slab interface and the columns. As the steel beam attempts to shorten due to the spread of local buckling across its depth, a compressive force, $N_{c}$, is generated in the slab in conjunction with the tensile force in the steel beam. The compressive force is transferred through shear in the studs, friction, bearing of the slab on the column face and a direct compression strut. At large lateral drift demands (SDR $\geq 4 \%$ ), the studs lose their shear capacity, and the last two load paths transfer the compressive force to the slab. This is particularly true for deep beams $\left(d_{b} \geq 700 \mathrm{~mm}\right)$ with low degree of composite action $(\eta<50 \%)$ as discussed in the next section.

The axial forces in the steel beam and slab are dependent on several parameters. These relate to the extent of beam axial shortening experienced in the absence of axial restraint, as well as the level of axial restraint. First and foremost, the magnitude of the tensile force in the steel beam is dependent on the susceptibility of the steel beam to local buckling across its depth. Since all three configurations studied herein are adequately braced laterally (see Table 1) and have nominally identical material properties, the difference in local buckling initiation in the beams is mostly governed by the beam's cross-section geometry. Lignos and Krawinkler (2011) found that $h_{b} / t_{w}$, in particular, largely influences local buckling initiation in intermediate to deep steel beams. The maximum $N_{s} / N_{p l}$ ratio increases with increasing $h_{b} / t_{w}$ (see Table 1). This is observed in Fig. 11 where specimen $S_{D}$ experiences the highest axial tensile force ratio.

Local buckling in shallow beams $\left(d_{b} \leq 500 \mathrm{~mm}\right)$ is localized in the lower portion of the beam due to the slab restraint on the top flange of the beam. This is also true for beams with high degree of composite action ( $\eta>80 \%$ ). Moreover, in the above cases, a compatibility compressive force occurs when the beam ends are pushed apart (see previous section). The compressive force alleviates the tensile force in the beam. Axial restraint is provided by the slab and the columns. In shallow beams, the axial restraint provided by the slab is relatively higher than that in deep beams. This is because the relative slab in-plane stiffness-to-beam axial stiffness is higher in shallow 
beams than in deep beams. On the other hand, the axial restraint provided by the columns is dependent on their flexural stiffness. Since columns sizes are strongly influenced by the SCWB ratio, the flexural stiffness of the columns normally increases with the beam depth. Therefore, the axial restraint provided by the columns is expected to be high in deep beams. As mentioned earlier, the level of axial restraint is higher at the interior joint than at the exterior joint due to (i) slab continuity and (ii) potentially stiffer columns at the interior joints. In the configurations considered in this paper, the same column cross-sections are used at the interior and exterior joints. Thus, we postulate that the difference in the axial restraint should not be substantial. The magnitude of the axial force in the beam also depends on its axial stiffness. However, for equal depth beams, a larger axial stiffness implies a stockier section with a lower susceptibility to local buckling. Based on the above, the major factor that determines the magnitude of $N_{S} / N_{p l}$ is the susceptibility of the beam to local buckling across its depth. The main controlling parameters in the examined cases are the beam depth, $d_{b}$ and the web local slenderness ratio, $h_{b} / t_{w}$.

\section{Shear Stud Hysteretic Response}

Seismic loads are transferred from the slab into the beam through shear in the stud connectors and friction at the beam-slab interface. In composite beams with shear studs as the weak link, early loss of composite action is likely to occur as a result of shear stud failure (Cordova and Deierlein 2005). Consequently, seismic loads are predominantly transferred to the column by bearing of the slab on the column face and a direct compression strut. This can lead to severe damage in the slab due to concrete spalling. Damage in the slab can be reduced if the integrity of the shear studs is maintained. From a design perspective, the shear studs at the slab-beam interface should sustain their load-carrying capacity. To this end, both ANSI/AISC 341-16 (AISC 2016b) and EN 1998-1 (CEN 2004a) recommend a 25\% reduction in the design shear resistance of the studs. However, past studies have shown that the performance of shear studs in composite steel MRFs is better than anticipated (Cordova and Deierlein 2005). An assessment of the stud degradation behavior is performed by obtaining the hysteretic stud shear-stud slip response in each of the composite beam specimens subjected to cyclic loading.

Figure 12 shows the hysteretic response of the west beam shear stud nearest to the interior joint. The shear studs in subassemblies $S_{D}, S_{I}$ and $S_{S}$, lose their load carrying capacity in sagging bending at $4 \%, 5 \%$ and $6 \%$ SDR respectively. At $4 \%$ lateral drift demands, the studs belonging to 
subassemblies $S_{I}$ and $S_{S}$ exhibit satisfactory behavior with little or no degradation, whereas that of specimen $S_{D}$ fails. This is despite the fact that all three specimens have the same slab configuration, number of studs and degree of composite action. The deeper the beam is, the higher the shear demand; hence, the more degradation in the shear studs connecting the beam to the slab. In subsystems, the stud shear force degradation is lower than that in the corresponding subassembly. Referring to Fig. 12(b), the shear stud in sub-system $S_{D}$ loses its capacity at 5\% SDR compared to $4 \%$ in the subassembly. Similarly, Figs. 12 (c and d) depict that the shear studs of sub-system $S_{I}$ and $S_{S}$ remain intact at the end of the analysis. In subassemblies, noticeable axial shortening in the beams tends to pry the beam away from the slab. Consequently, the shear studs that restrain the beam against axial shortening are subjected to an additional shear demand. The additional demand increases the stud shear force in the sagging bending regions and reduces it in the hogging bending regions. A higher rate of strength degradation is observed in studs belonging to subassemblies when compared to those in sub-systems. Initially, the hysteretic behavior of the shear studs coincides as shown in Figure 12. Once beam axial shortening initiates, a discrepancy in the behavior of the shear studs is observed.

In EN 1994-1-1 (CEN 2004b), ductile shear connectors are defined as the ones with a characteristic deformation capacity, $\delta_{u k}=6 \mathrm{~mm}$ at $90 \%$ of the ultimate shear resistance (Bärtschi 2005). Hence, the headed shear studs, with which the non-linear springs are calibrated, are ductile as per EN 1994-1-1 (CEN 2004b). The maximum stud slip demands at 4\% SDR are $11 \mathrm{~mm}, 8 \mathrm{~mm}$ and $2 \mathrm{~mm}$ for sub-systems $\mathrm{S}_{\mathrm{D}}, \mathrm{S}_{\mathrm{I}}$ and $\mathrm{S}_{\mathrm{S}}$ respectively. Out of the three sub-systems, only the stud slip demand in $S_{S}$, does not exceed the characteristic deformation capacity of ductile shear connectors. However, the stud slip in all cases is within $6 \mathrm{~mm}\left(4 \mathrm{~mm}, 2 \mathrm{~mm}\right.$ at $1 \mathrm{~mm}$ for $S_{D}, S_{I}$ and $\mathrm{S}_{\mathrm{S}}$ respectively) at modest lateral drift demands (i.e., 2\%) characteristic of a design-basis earthquake corresponding to a probability of exceedance of $10 \%$ in 50 years. Additionally, a higher degree of composite action would decrease the shear demand on the studs. Vis-à-vis the above discussion, the general consensus is that for shallow to intermediate composite beams $\left(d_{b} \leq\right.$ $500 \mathrm{~mm}$ ) no reduction in the shear resistance of studs is imperative as long as ductile shear studs are used. For deeper beams, a reduction in the shear resistance of studs is deemed reasonable.

\section{Influence of Loading Protocol}


In the previous sections, the hysteretic behavior of the 2-bay sub-systems was examined under a symmetric cyclic lateral-loading protocol. However, this protocol overestimates the seismic demand in the frame and subsequently the cyclic deterioration of the beams (FEMA 2009), if limit states associated with structural collapse are of interest. In that respect, collapse-consistent protocols (Krawinkler 2009; Maison and Speicher 2016; Suzuki and Lignos 2019) are more realistic for estimating seismic demands in structural components at limit states associated with earthquake-induced collapse (Lignos et al. 2011). In order to further comprehend the differences in the hysteretic behavior of sub-systems subjected to symmetric cyclic and collapse-consistent protocols, sub-system $S_{D}$ is subjected to a collapse consistent-loading protocol derived according to Suzuki and Lignos (2019). The protocol consists of three phases and represents a near-fault ground motion with a low probability of occurrence. Each phase includes a few inelastic cycles followed by a large monotonic push. The asymmetric drift demand replicates the characteristic "ratcheting" behavior of frame structures prior to collapse (Lignos et al. 2011).

Referring to Fig. 13(a), at 5\% SDR, the west beam hogging moment $\left(M_{b, W e s t, C 2}\right.$ at the face of column $\mathrm{C} 2$ ) degrades by less than $5 \%$ under the collapse-consistent protocol. On the other hand, the beam's flexural strength degradation is more than $20 \%$ under the symmetric cyclic loading protocol. Due to ratcheting of the frame, no degradation in the sagging moment occurs. Local buckling is minor and is localized in the lower portion of the steel beam. This explains the marginally lesser amount of axial shortening $(4 \mathrm{~mm})$ experienced in the west beam under the collapse-consistent protocol compared to the symmetric cyclic protocol $(7 \mathrm{~mm})$ as depicted in Fig. 13(c). The beam experiences greater cyclic degradation under the symmetric cyclic loading protocol than the collapse-consistent loading protocol due to the larger number of inelastic cycles in the former. This agrees with prior observations from large- and full-scale physical testing (Elkady and Lignos 2018b; Suzuki and Lignos 2015). Figure 13(b) shows that the peak panel zone shear distortion is higher under the collapse-consistent protocol $\left(10 \gamma_{y}\right)$ than that observed under the symmetric cyclic protocol $\left(6 \gamma_{y}\right)$. The ratcheting response is mostly attributed to the asymmetric drift demand. Finally, the hysteretic behavior of the west shear stud nearest to the interior joint is compared in Fig. 13(d). The stud loss of shear resistance occurs at 5\% SDR, regardless of the employed lateral loading protocol. Prior to 5\% SDR, the seismic shear demand in the studs is similar under both loading conditions. Furthermore, since the shear strength degradation due to 
cyclic loading is not significant (see Fig. 2), the rate of degradation is nearly the same in both cases.

\section{Limitations and Assumptions}

Considering the modeling assumptions and simplifications discussed herein, it is worth highlighting the following limitations: (i) the CFE model is not capable of capturing fracture in the beam-to-column connection and ductile tearing due to extensive local buckling; (ii) bond slip between the reinforcement and concrete is not modeled explicitly; (iii) no separation is allowed between the concrete slab and the steel deck; and (iv) concrete spalling due to crushing is not explicitly considered. Despite these shortcomings, the modeling approach is deemed capable of simulating the physical mechanisms associated with the slab restraint and the overall cyclic behavior of connections in sub-systems and subassemblies.

\section{Conclusions}

This paper investigates the effects of the axial restraint provided by the slab and the columns (frame continuity) on the hysteretic behavior of typical beam-to-column connections with a composite floor slab. First, a detailed continuum finite element (CFE) model is proposed and validated with available experimental data. The CFE model explicitly captures the interaction between the slab and the beam, as well as the cyclic degradation of the shear stud connectors. Next, the CFE approach is extended to model two-bay sub-systems with three different beam depths representative of both North American and European design practice. The effects of the axial restraint and framing action are examined by comparing the behavior of sub-systems with that of the corresponding subassemblies. The major findings are summarized below:

- Qualitatively, the panel zone contribution to the story drift is higher in the sub-system interior joints than in the corresponding cruciform subassembly joints. This is attributed to the lower rate of the beams' flexural strength degradation. In the sub-system exterior joints, the panel zone contribution to the story drift is dependent on the direction of loading: under sagging excursions, the panel zone contribution to the story drift may reach up to $40 \%$, despite the panel zone design compliance to the ANSI/AISC-341-16 seismic provisions (AISC 2016b). On the other hand, under hogging bending, the beam deformation dominates the lateral drift demand. 
- Under symmetric-cyclic lateral loading, beams in two-bay sub-systems may experience up to $25 \%$ less flexural strength degradation than their subassembly counterparts. This is particularly evident in deep and slender beams. In sub-systems, the local buckles in the beams are straightened due to the axial restraint provided by the floor slab and the columns. It is observed that the straightening is more prominent under sagging bending than hogging bending. This leads to the underestimation of the probable maximum moment $M_{f}$ (by up to $25 \%$ ), even in deep beams where the flexural strength amplification due to composite action is fairly small. This issue may be compelling for sizing columns and estimating the shear demand in panel zones of capacity-designed steel MRFs.

- The interior joint panel zones in sub-systems experience up to $15 \%$ higher shear distortion than their subassembly counterparts. Their hysteretic behavior is symmetric. On the other hand, exterior joint panel zones in sub-systems exhibit a distinct asymmetric response due to the different shear demands under sagging and hogging bending. The difference in shear demands is attributed to three underlying mechanisms namely: (i) composite action, (ii) the slab restraint against top flange local buckling; and (iii) the axial restraint provided by the slab and the columns. The CFE analysis reveals that panel zones in sub-systems may experience a shear distortion higher than the anticipated value for which they were designed (i.e., $4 \gamma_{y}$ ). Nonetheless, premature fracture due to panel zone shear distortion is not expected as the maximum shear distortion is lower than $10 \gamma_{y}$.

- Subassembly beams may experience severe axial shortening (up to $50 \mathrm{~mm}$ at $6 \% \mathrm{SDR}$ ). The degree of axial shortening is higher in deep beams with high web slenderness ratios close to the current compactness limits of highly ductile members according to the ANSI/AISC 34116 seismic provisions (AISC 2016b). On the other hand, beam axial shortening observed in sub-systems is considerably less (up to $7 \mathrm{~mm}$ at $6 \%$ SDR) than that observed in subassemblies (up to $50 \mathrm{~mm}$ at $6 \% \mathrm{SDR}$ ). It is inferred that axial shortening is overestimated in subassembly experiments commonly used in experimental earthquake engineering. In real buildings, beam axial shortening is much lower, akin to that in sub-systems.

- Axial forces develop in composite beams as a consequence of the axial restraint. At the inflection point, the axial tensile force in the steel beam's cross-section may reach slightly higher than $15 \% N_{p l}$ at $6 \% \mathrm{SDR}$. The tensile force magnitude is dependent on the susceptibility of the beam to local buckling across its depth, as well as on the level of axial restraint. The 
former is particularly high in deep and slender beams $\left(d_{b} \geq 700 \mathrm{~mm}\right)$ with low degree of composite action $(\eta<50 \%)$. The latter depends on the relative in-plane slab-to-beam axial stiffness (higher in shallow beams) and the flexural stiffness of the columns (higher in deep beam sub-systems). This issue should be examined in conjunction with the catenary action imposed to the steel girders of a beam-to-column connection due to column axial shortening (Elkady and Lignos 2018b; Suzuki and Lignos 2015).

- Comparisons between the hysteretic behavior of shear studs in sub-systems and subassemblies suggest that the shear force degradation in the latter is higher than that of the former. This is due to axial shortening in the beam that tends to pry the beam away from the slab. The CFE models indicate that higher stud shear force degradation occurs in sub-systems with deep beams than in those with intermediate to shallow beams. However, at $2 \%$ lateral drift demands associated with a design-basis earthquake, the stud slip demand remains within the characteristic deformation capacity of ductile shear connectors (6mm) according to EN 19941-1 (CEN 2004b). At 4\% lateral drift demand, the slip demand exceeded $6 \mathrm{~mm}$ in all but the sub-system with shallow beams. For shallow beams or beams with high degree of composite action (i.e., above $80 \%$ ), it seems reasonable to omit the $25 \%$ reduction in shear strength of the studs required in both ANSI/AISC 341-16 (AISC 2016b) and EN 1998-1 (CEN 2004a).

- The response of sub-systems under collapse-consistent lateral load protocols suggests that beam flexural strength deterioration and axial shortening is inconsequential compared to that under a symmetric loading history. Conversely, the panel zone shear distortion may reach $10 \gamma_{y}$ in exterior joints. The shear stud hysteretic behavior does not seem to be influenced by the employed loading history.

\section{Acknowledgments}

This study is based on work supported by the Swiss National Science Foundation (Project No. 200021_169248). The financial support is gratefully acknowledged. Any opinions expressed in the paper are those of the authors and do not necessarily reflect the views of sponsors.

\section{Notation}

The following symbols are used in this paper:

$A_{g} \quad=$ cross-sectional area of the steel beam

$D^{-} \quad=$ rate of cyclic deterioration of the shear stud when the slab is under tension 


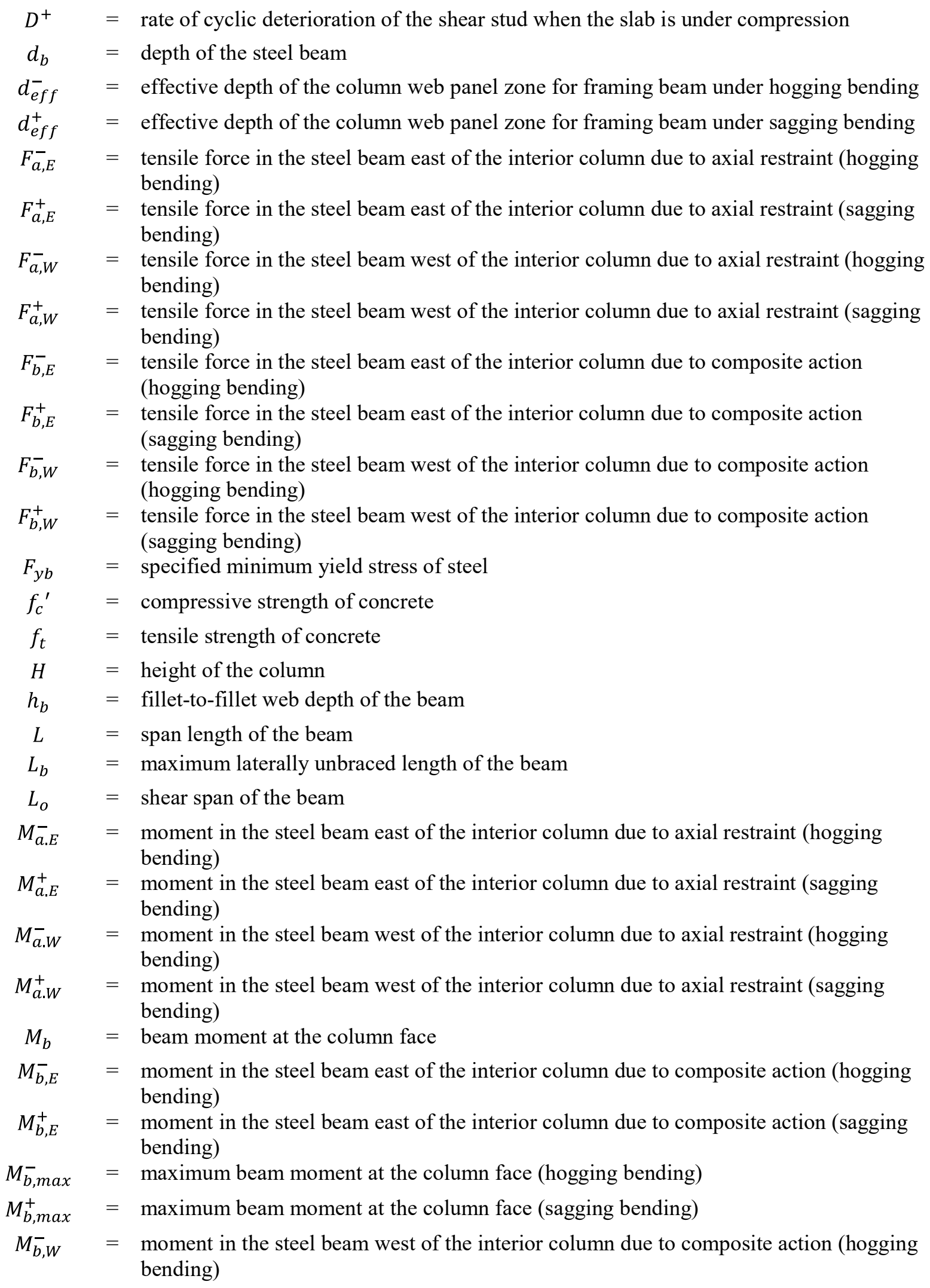




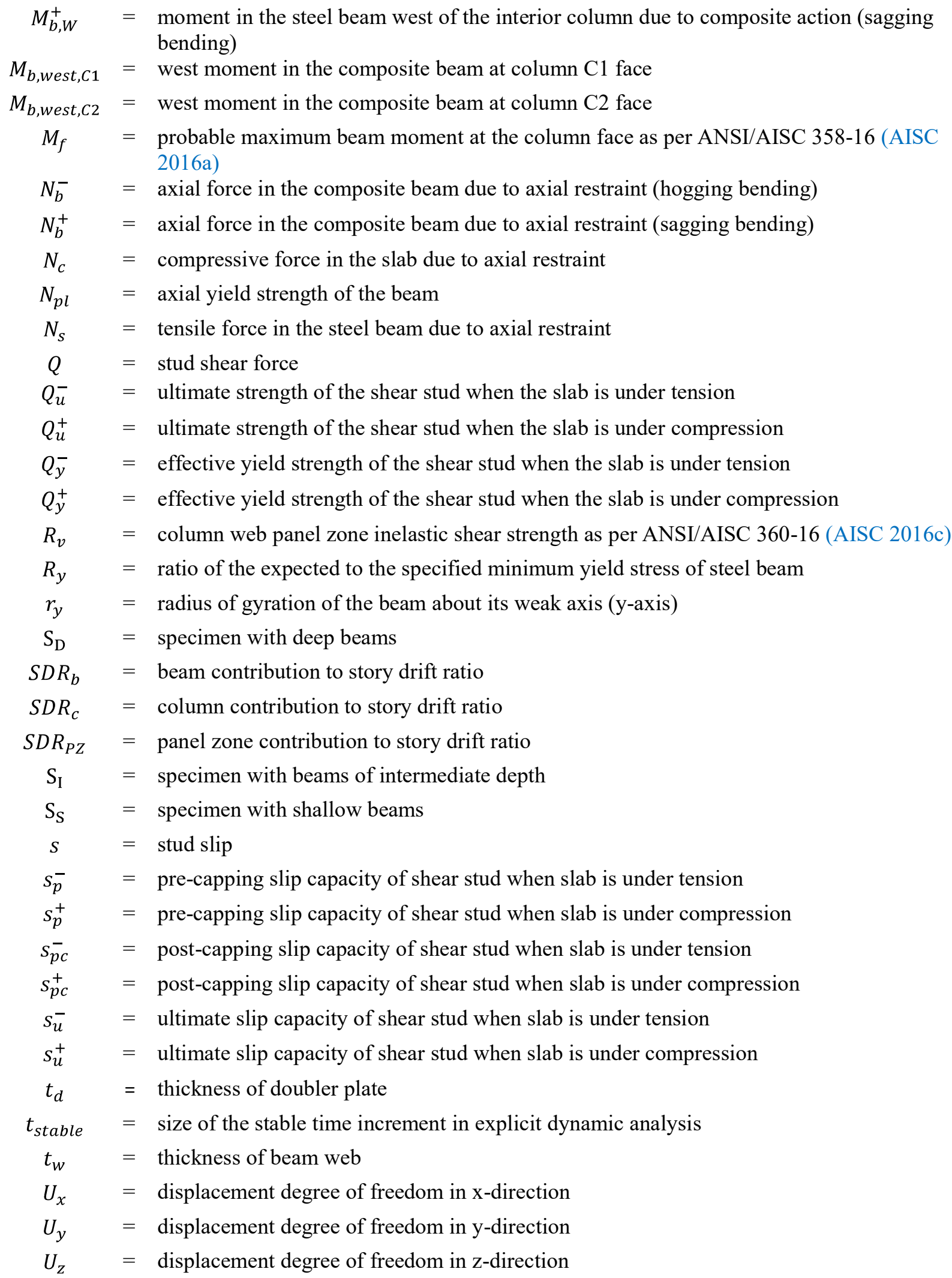




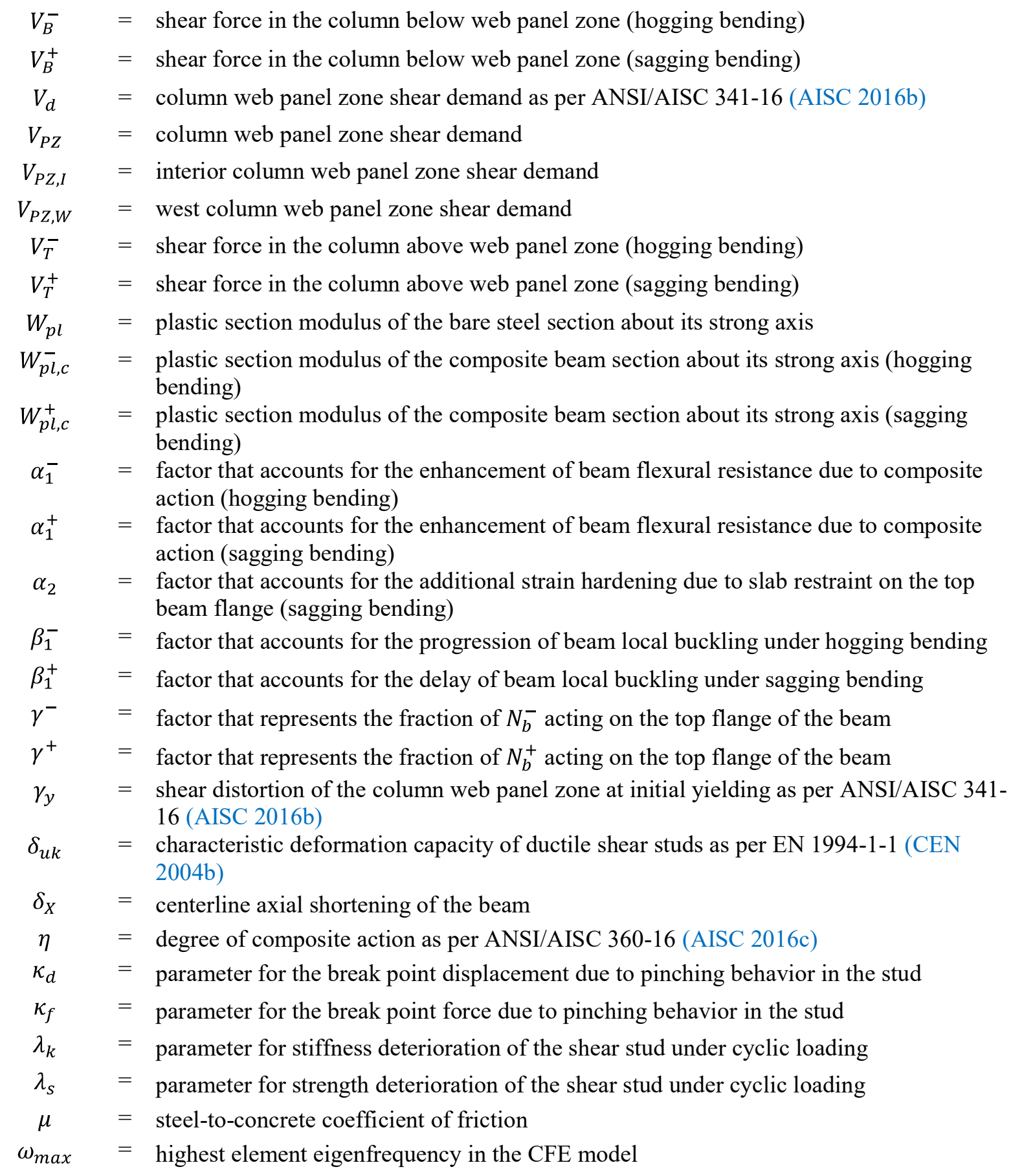

675 Abaqus. (2014). Abaqus 6.14 Documentation. Dassault Systemès Simulia Corp., RI, USA. 
AISC. (2016a). Prequalified connections for special and intermediate steel moment frames for seismic applications, ANSI/AISC 358-16. American Institute for Steel Construction, Chicago, IL.

AISC. (2016b). Seismic provisions for structural steel buildings, ANSI/AISC 341-16. American Institute for Steel Construction, Chicago, IL.

AISC. (2016c). Specification for structural steel buildings, ANSI/AISC 360-16. American Institute for Steel Construction, Chicago, IL.

Alashker, Y., El-Tawil, S., and Sadek, F. (2010). "Progressive collapse resistance of steel-concrete composite floors." Journal of Structural Engineering, 136(10), 1187-1 196.

Amadio, C., and Fragiacomo, M. (1993). "A finite element model for the study of creep and shrinkage effects in composite beams with deformable shear connections." Costruzioni Metalliche, 4, 213-228.

Ayoub, A. (2005). "A force-based model for composite steel-concrete beams with partial interaction." Journal of Constructional Steel Research, 61(3), 387-414.

Ayoub, A., and Filippou, F. C. (2000). "Mixed Formulation of Nonlinear Steel-Concrete Composite Beam Element." Journal of Structural Engineering, 126(3), 371-381.

Bärtschi, R. (2005). Load Bearing Behaviour of Composite Beams in Low Degrees of Partial Shear Connection. IBK Bericht, Swiss Federal Institute of Technology Zürich, Zürich.

Baskar, K., Shanmugam, N. E., and Thevendran, V. (2002). "Finite-Element Analysis of SteelConcrete Composite Plate Girder." Journal of Structural Engineering, 128(9), 1158-1168.

Bursi, O. S., and Ballerini, M. (1996). "Behavior of a steel-concrete composite substructure with full and partial shear connection." Proceedings of 11th World Conference on Earthquake Engineering, Acapulco, Mexico.

Bursi, O. S., and Gramola, G. (1999). "Behaviour of headed stud shear connectors under lowcycle high amplitude displacements." Materials and structures, 32(4), 290-297.

Bursi, O. S., and Jaspart, J. P. (1998). "Basic issues in the finite element simulation of extended end plate connections." Computers \& Structures, 69(3), 361-382.

Bursi, O. S., Sun, F.-F., and Postal, S. (2005). "Non-linear analysis of steel-concrete composite frames with full and partial shear connection subjected to seismic loads." Journal of Constructional Steel Research, 61(1), 67-92.

Carreira, D. J., and Chu, K.-H. (1985). "Stress-strain relationship for plain concrete in compression." Journal Proceedings, 797-804.

CEN. (2004a). EN 1998-1: Eurocode 8: Design of structures for earthquake resistance - Part 1: General rules, seismic actions and rules for buildings. European Committee for Standardization, Brussels, Belgium.

CEN. (2004b). EN 1994-1-1: Eurocode 4: Design of composite steel and concrete structures Part 1-1: General rules and rules for buildings. European Committee for Standardization, Brussels, Belgium.

Cheng, C.-T., and Chen, C.-C. (2005). "Seismic behavior of steel beam and reinforced concrete column connections." Journal of Constructional Steel Research, 61(5), 587-606.

Chung, W. J., Cho, J. W., and Belytschko, T. (1998). "On the dynamic effects of explicit FEM in sheet metal forming analysis." Engineering Computations, 15(6), 750-776.

Civjan, S. A., and Singh, P. (2003). "Behavior of shear studs subjected to fully reversed cyclic loading." Journal of Structural Engineering, 129(11), 1466-1474.

Civjan, S., Engelhardt, M., and Gross, J. (2001). "Slab effects in SMRF retrofit connection tests." Journal of Structural Engineering, 127(3), 230-237. 
Clifton, C., Bruneau, M., MacRae, G., Leon, R., and Fussell, A. (2011). "Steel structures damage from the Christchurch earthquake series of 2010 and 2011." Bulletin of the New Zealand Society for Earthquake Engineering, 44(4), 297-318.

Cordova, P. P., and Deierlein, G. (2005). Validation of the seismic performance of composite RCS frames: Full-scale testing, analytical modeling, and seismic design. The John A. Blume Earthquake Engineering Center, Stanford University, Stanford, CA.

Deierlein, G. G., Reinhorn, A. M., and Willford, M. R. (2010). Nonlinear structural analysis for seismic design. NEHRP Seismic Design Technical Brief No. 4, National Institute of Standards and Technology, Gaithersburg, MD, 1-36.

Del Carpio, M., Mosqueda, G., and Lignos, D. (2014). Hybrid simulation of the seismic response of a steel moment frame building structure through collapse. Technical Report, Multidisciplinary Center for Earthquake Engineering Research, University at Buffalo.

Donahue, S., Engelhardt, M., Clayton, P., Williamson, E., and Helwig, T. (2017). "Role of the Floor System in the Cyclic Response of Composite Steel Gravity Framing." Spring Creek Ranch, Jackson Hole, USA.

El Jisr, H., Elkady, A., and Lignos, D. G. (2019). "Composite steel beam database for seismic design and performance assessment of composite-steel moment-resisting frame systems." Bulletin of Earthquake Engineering, 17(6), 3015-3039.

Elkady, A., and Lignos, D. G. (2014). "Modeling of the composite action in fully restrained beamto-column connections: implications in the seismic design and collapse capacity of steel special moment frames." Earthquake Engineering \& Structural Dynamics, 43(13), 19351954.

Elkady, A., and Lignos, D. G. (2015a). "Effect of gravity framing on the overstrength and collapse capacity of steel frame buildings with perimeter special moment frames." Earthquake Engineering \& Structural Dynamics, 44(8), 1289-1307.

Elkady, A., and Lignos, D. G. (2015b). "Analytical investigation of the cyclic behavior and plastic hinge formation in deep wide-flange steel beam-columns." Bulletin of Earthquake Engineering, 13(4), 1097-1118.

Elkady, A., and Lignos, D. G. (2018a). "Improved seismic design and nonlinear modeling recommendations for wide-flange steel columns." Journal of Structural Engineering, 144(9), 04018162.

Elkady, A., and Lignos, D. G. (2018b). "Full-scale testing of deep wide-flange steel columns under multiaxis cyclic loading: Loading sequence, boundary effects, and lateral stability bracing force demands." Journal of Structural Engineering, 144(2), 04017189.

El-Tawil, S., and Deierlein, G. G. (2001). "Nonlinear Analysis of Mixed Steel-Concrete Frames. I: Element Formulation." Journal of Structural Engineering, 127(6), 647-655.

Engelhardt, M., Venti, M., Fry, G., Jones, S., and Holliday, S. (2000). Behavior and design of radius-cut reduced beam section connections. SAC Joint Venture.

FEMA. (2000a). State of the art report on connection performance. Federal Emergency Management Agency, Washington D.C.

FEMA. (2000b). Recommended seismic design criteria for new steel moment-frame buildings.

FEMA. (2009). Effects of strength and stiffness degradation on seismic response. Federal Emergency Management Agency, Washington D.C.

Gattesco, N. (1999). "Analytical modeling of nonlinear behavior of composite beams with deformable connection." Journal of Constructional Steel Research, 52(2), 195-218. 
Genikomsou, A. S., and Polak, M. A. (2015). "Finite element analysis of punching shear of concrete slabs using damaged plasticity model in ABAQUS." Engineering Structures, 98, $38-48$.

Goto, Y., Kumar, G. P., and Kawanishi, N. (2010). "Nonlinear finite-element analysis for hysteretic behavior of thin-walled circular steel columns with in-filled concrete." Journal of structural engineering, 136(11), 1413-1422.

Herrera, R. A., Ricles, J. M., and Sause, R. (2008). "Seismic performance evaluation of a largescale composite MRF Using pseudodynamic testing." Journal of Structural Engineering, 134(2), 279-288.

Ibarra, L. F., Medina, R. A., and Krawinkler, H. (2005). "Hysteretic models that incorporate strength and stiffness deterioration." Earthquake Engineering \& Structural Dynamics, 34(12), 1489-1511.

Johansson, M., and Gylltoft, K. (2002). "Mechanical behavior of circular steel-concrete composite stub columns." Journal of structural engineering, 128(8), 1073-1081.

Kim, K. D., and Engelhardt, M. D. (2002). "Monotonic and cyclic loading models for panel zones in steel moment frames." Journal of Constructional Steel Research, 58(5-8), 605-635.

Kim, S.-Y., and Lee, C.-H. (2017). "Seismic retrofit of welded steel moment connections with highly composite floor slabs." Journal of Constructional Steel Research, 139, 62-68.

Kolwankar, S., Kanvinde, A., Kenawy, M., Lignos, D., and Kunnath, S. (2018). "Simulating local buckling-induced softening in steel members using an equivalent nonlocal material model in displacement-based fiber elements." Journal of Structural Engineering, 144(10), 04018192.

Krawinkler, H. (2009). "Loading histories for cyclic tests in support of performance assessment of structural components." The 3rd International Conference on Advances in Experimental Structural Engineering, San Francisco.

Kwasniewski, L., Stojadinović, B., and Goel, S. C. (2002). Local and lateral-torsional buckling of wide-flange beams. SAC Joint Venture.

Landolfo, R., D’Aniello, M., Costanzo, S., Tartaglia, R., Stratan, A., Dubina, D., Vulcu, C., Maris, C., Zub, C., Da Silva, L., Rebelo, C., Augusto, H., Shahbazian, A., Gentili, F., Jaspart, J.P., Demonceau, J.-F., Van Hoang, L., Elghazouli, A., Tsitos, A., Vassart, O., Nunez, E. M., Dehan, V., and Hamreza, C. (2018). European pre-QUALified steel JOINTS (EQUALJOINTS). European Commission, Brussels, Belgium.

Lee, J., and Fenves. (1998). "Plastic-Damage Model for Cyclic Loading of Concrete Structures." Journal of Engineering Mechanics, 124(8), 892-900.

Lemaitre, J., and Chaboche, J.-L. (1994). Mechanics of solid materials. Cambridge university press.

Leon, R. T., Hajjar, J. F., and Gustafson, M. A. (1998). "Seismic response of composite momentresisting connections. I: Performance." Journal of Structural Engineering, 124(8), 868876.

Liang, Q. Q., Uy, B., Bradford, M. A., and Ronagh, H. R. (2005). "Strength Analysis of SteelConcrete Composite Beams in Combined Bending and Shear." Journal of Structural Engineering, 131(10), 1593-1600.

Lignos, D. G., Krawinkler, H., and Whittaker, A. S. (2011). "Prediction and validation of sidesway collapse of two scale models of a 4-story steel moment frame." Earthquake Engineering \& Structural Dynamics, 40(7), 807-825. 
Lignos, D., and Krawinkler, H. (2011). "Deterioration modeling of steel components in support of collapse prediction of steel moment frames under earthquake loading." Journal of Structural Engineering, 137(11), 1291-1302.

Liu, J., and Astaneh-Asl, A. (2004). "Moment-rotation parameters for composite shear tab connections." Journal of Structural Engineering, 130(9), 1371-1380.

Lubliner, J., Oliver, J., Oller, S., and Oñate, E. (1989). "A plastic-damage model for concrete." International Journal of Solids and Structures, 25(3), 299-326.

MacRae, G. A., and Clifton, G. C. (2015). "Research on seismic performance of steel structures." Auckland, New Zealand.

MacRae, G. A., Hobbs, M., Bull, D., Chaudhari, T., Leon, R., Clifton, G. C., and Chase, J. G. (2013). "Slab Effects on Beam-Column Subassemblies-Beam Strength and Elongation Issues." Palm Cove, Queensland, Australia.

Mahin, S. A. (1998). "Lessons from damage to steel buildings during the Northridge earthquake." Engineering Structures, Innovations in Stability Concepts and Methods for Seismic Design in Structural Steel, 20(4), 261-270.

Maison, B. F., and Speicher, M. S. (2016). "Loading protocols for ASCE 41 backbone curves." Earthquake Spectra, 32(4), 2513-2532.

Matsumura, T., and Mizuno, E. (2007). "3-D FEM analyses on internal state inside the concrete filled steel tubular columns subjected to flexural deformation under axial loading." Journal of Structural Engineering, 53, 75-83.

Mehanny, S. S., and Deierlein, G. (2000). Modeling of Assessment of Seismic Performance of Composite Frames with Reinforced Concrete Columns and Steel Beams. John A. Blume Earthquake Engineering Center Report, Stanford University, Stanford, CA.

Okazaki, T., Lignos, D. G., Midorikawa, M., Ricles, J. M., and Love, J. (2013). "Damage to steel buildings observed after the 2011 Tohoku-Oki earthquake." Earthquake spectra, 29(S1), S219-S243.

PEER/ATC. (2010). Modeling and acceptance criteria for seismic design and analysis of tall buildings. Applied Technology Council (ATC), Redwood City, CA.

Prior, A. M. (1994). "Applications of implicit and explicit finite element techniques to metal forming." Journal of Materials Processing Technology, 45(1-4), 649-656.

Qi, L., Paquette, J., Eatherton, M., Leon, R., Bogdan, T., Popa, N., and Nunez, E. (2018). "Analysis of Fracture Behavior of Large Steel Beam-Column Connections." Proceedings of the 12th International Conference on Advances in Steel-Concrete Composite Structures. ASCCS 2018, Editorial Universitat Politècnica de València, 521-526.

Rassati, G. A., Leon, R. T., and Noè, S. (2004). "Component Modeling of Partially Restrained Composite Joints under Cyclic and Dynamic Loading." Journal of Structural Engineering, 130(2), 343-351.

Rex, C. O., and Easterling, W. S. (2000). "Behavior and modeling of reinforced composite slab in tension." Journal of Structural Engineering, 126(7), 764-771.

Ricles, J. M., Fisher, J. W., Lu, L.-W., and Kaufmann, E. J. (2002). "Development of improved welded moment connections for earthquake-resistant design." Journal of Constructional Steel Research, North American Special Issue, 58(5), 565-604.

SAC Joint Venture. (1997). "Protocol for fabrication, inspection, testing, and documentation of beam-column connection tests and other experimental specimens." Rep. No. SAC/BD-97, 2. 
Salari, M. R., and Spacone, E. (2001). "Finite element formulations of one-dimensional elements with bond-slip." Engineering Structures, 23(7), 815-826.

Schafer, B., Easterling, W. S., Sabelli, R., Eatherton, M. R., and Hajjar, J. F. (2019). Steel Diaphragm Innovation Initiative Workshop Report. The Cold-Formed Steel Research Consortium (CFSRC).

Selamet, S., and Garlock, M. (2010). "Guidelines for modeling three dimensional structural connection models using finite element methods." International Symposium: Steel Structures: Culture \& Sustainability.

Shin, S., and Engelhardt, M. D. (2013). "Cyclic performance of deep column moment frames with weak panel zones." Advances in structural engineering and mechanics (ASEM13), Jeju, Korea.

Sjaarda, M., Walbridge, S., and West, J. S. (2018). "Assessment of shear connection through composite beam modeling." Transportation Research Record, 0361198118781685.

Sousa, A. de C., and Lignos, D. (2018). On the inverse problem of classic nonlinear plasticity models: An application to cyclically loaded structural steels. Ecole Polytechnique Federale de Lausanne, Lausanne, Switzerland.

Sumner, E. A., and Murray, T. M. (2002). "Behavior of extended end-plate moment connections subject to cyclic loading." Journal of Structural Engineering, 128(4), 501-508.

Suzuki, A., and Kimura, Y. (2019). "Cyclic behavior of component model of composite beam subjected to fully reversed cyclic loading." Journal of Structural Engineering, 145(4), 04019015.

Suzuki, Y., and Lignos, D. (2018). "Fiber-based model for earthquake-induced collapse simulation of steel frame buildings." Proceedings of the 11th US National Conference on Earthquake Engineering.

Suzuki, Y., and Lignos, D. G. (2015). "Large scale collapse experiments of wide flange steel beamcolumns." 8th International Conference on Behavior of Steel Structures in Seismic Areas (STESSA).

Suzuki, Y., and Lignos, D. G. (2019). "Development of collapse-consistent loading protocols for experimental testing of steel columns." Earthquake Engineering \& Structural Dynamics.

Tartaglia, R., D'Aniello, M., Rassati, G. A., Swanson, J. A., and Landolfo, R. (2018). "Full strength extended stiffened end-plate joints: AISC vs recent European design criteria." Engineering Structures, 159, 155-171.

Tremblay, R., Tchebotarev, N., and Filiatrault, A. (1997). "Seismic performance of RBS connections for steel moment resisting frames: Influence of loading rate and floor slab." Proceedings of Stessa.

Tsitos, A., Bravo-Haro, M. A., and Elghazouli, A. Y. (2018). "Influence of deterioration modelling on the seismic response of steel moment frames designed to Eurocode 8." Earthquake Engineering \& Structural Dynamics, 47(2), 356-376.

Uang, C.-M., Yu, Q.-S., Noel, S., and Gross, J. (2000). "Cyclic testing of steel moment connections rehabilitated with RBS or welded haunch." Journal of Structural Engineering, 126(1), 57 68.

Voce, E. (1948). "The relationship between stress and strain for homogeneous deformation." Journal of the Institute of Metals, 74, 537-562.

Yamada, S., Satsukawa, K., Kishiki, S., Shimada, Y., Matsuoka, Y., and Suita, K. (2009). "Elastoplastic behavior of panel zone in beam to external column connection with concrete slab." Journal of Structural and Construction Engineering, AIJ, 74(664), 1841-1849. 
Young, B. W. (1972). "Residual stresses in hot-rolled members." Paris, 1-30.

904 Zandonini, R., and Bursi, O. S. (2002). "Cyclic behavior of headed stud shear connectors." Composite Construction in Steel and Concrete IV, Proceedings.

Zerbe, H., and Durrani, A. (1989). "Seismic response of connections in two- bay R/C frame subassemblies." Journal of Structural Engineering, 115(11), 2829-2844.

Zhang, X., Ricles, J., Lu, L.-W., and Fisher, J. (2004). Development of seismic guidelines for deepcolumn steel moment connections. ATLSS Report, Lehigh University.

Zhou, F., Mosalam, K. M., and Nakashima, M. (2007). "Finite-element analysis of a composite frame under large lateral cyclic loading." Journal of Structural Engineering, 133(7), 10181026. 
925 Table 1. CFE virtual testing matrix

\begin{tabular}{|c|c|c|c|c|c|c|c|c|c|c|c|c|}
\hline & \multicolumn{6}{|c|}{ Beam } & \multicolumn{2}{|c|}{ Column } & \multicolumn{2}{|c|}{$R_{v} / V_{d}$} & \multicolumn{2}{|c|}{$S C W B$} \\
\hline & Section & $\begin{array}{c}d_{b} \\
{[\mathrm{~mm}]}\end{array}$ & $\frac{h_{b}}{t_{w}}$ & $\frac{b_{f}}{2 t_{f}}$ & $\frac{L_{o}}{d_{b}}$ & $\frac{L_{b}}{r_{y}}$ & Section & $\begin{array}{c}t_{d} \\
{[\mathrm{~mm}]}\end{array}$ & Int. & Ext. & Int. & Ext. \\
\hline $\mathrm{S}_{\mathrm{D}}^{a, b}$ & W36x 150 & 911 & 51.9 & 6.4 & 4.5 & 26.7 & W27x194 & 12.7 & 1.1 & 1.2 & 1.5 & 2.81 \\
\hline $\mathrm{S}_{\mathrm{I}}^{a}$ & W21x122 & 551 & 31.3 & 6.5 & 7.6 & 22.6 & W24x162 & 22.2 & 1.2 & 1.2 & 2.1 & 3.81 \\
\hline $\mathrm{Ss}^{a}$ & W16x45 & 409 & 41.1 & 6.2 & 10.5 & 42.0 & W14x132 & 6.4 & 1.2 & 1.9 & 3.4 & 5.98 \\
\hline $\begin{array}{l}a=\mathrm{C} \\
b=\mathrm{C} \\
R_{v}=\mathrm{I} \\
V_{d}=\mathrm{I} \\
S C W 1\end{array}$ & $\begin{array}{l}\text { lic symmet } \\
\text { apse consi } \\
\text { lel zone in } \\
\text { tel zone sh } \\
\text { Strong-co }\end{array}$ & $\begin{array}{l}\text { ic load } \\
\text { tent loc } \\
\text { lastic s } \\
\text { ar dem } \\
\text { lumn-w }\end{array}$ & $\begin{array}{l}\text { ng his } \\
\text { ding p } \\
\text { lear stı } \\
\text { and (A } \\
\text { eak-be }\end{array}$ & $\begin{array}{l}\text { ngth } \\
\text { C } 20 \\
\text { n rati }\end{array}$ & 6b) & 16c) & $\begin{array}{l}\text { (SAC Jo } \\
\text { s (Suzuki }\end{array}$ & $\mathrm{tVer}$ & re 19 & & & \\
\hline
\end{tabular}


927 Table 1. Maximum west beam moment degradation at 6\% SDR

\begin{tabular}{clcccc}
\hline & & \multicolumn{2}{c}{ Sagging Bending } & \multicolumn{2}{c}{ Hogging Bending } \\
\cline { 3 - 6 } & & $W I$ & $W E$ & $W I$ & $W E$ \\
\hline \multirow{2}{*}{$\mathrm{S}_{\mathrm{D}}$} & Subassembly & $34 \%$ & NA & $44 \%$ & NA \\
& Sub-system & $12 \%$ & $4 \%$ & $23 \%$ & $38 \%$ \\
\hline \multirow{2}{*}{$\mathrm{S}_{\mathrm{I}}$} & Subassembly & $3 \%$ & NA & $6 \%$ & NA \\
& Sub-system & $0 \%$ & $0 \%$ & $6 \%$ & $6 \%$ \\
\hline \multirow{2}{*}{$\mathrm{S}_{\mathrm{S}}$} & Subassembly & $16 \%$ & NA & $9 \%$ & NA \\
& Sub-system & $0 \%$ & $0 \%$ & $10 \%$ & $19 \%$ \\
\hline
\end{tabular}




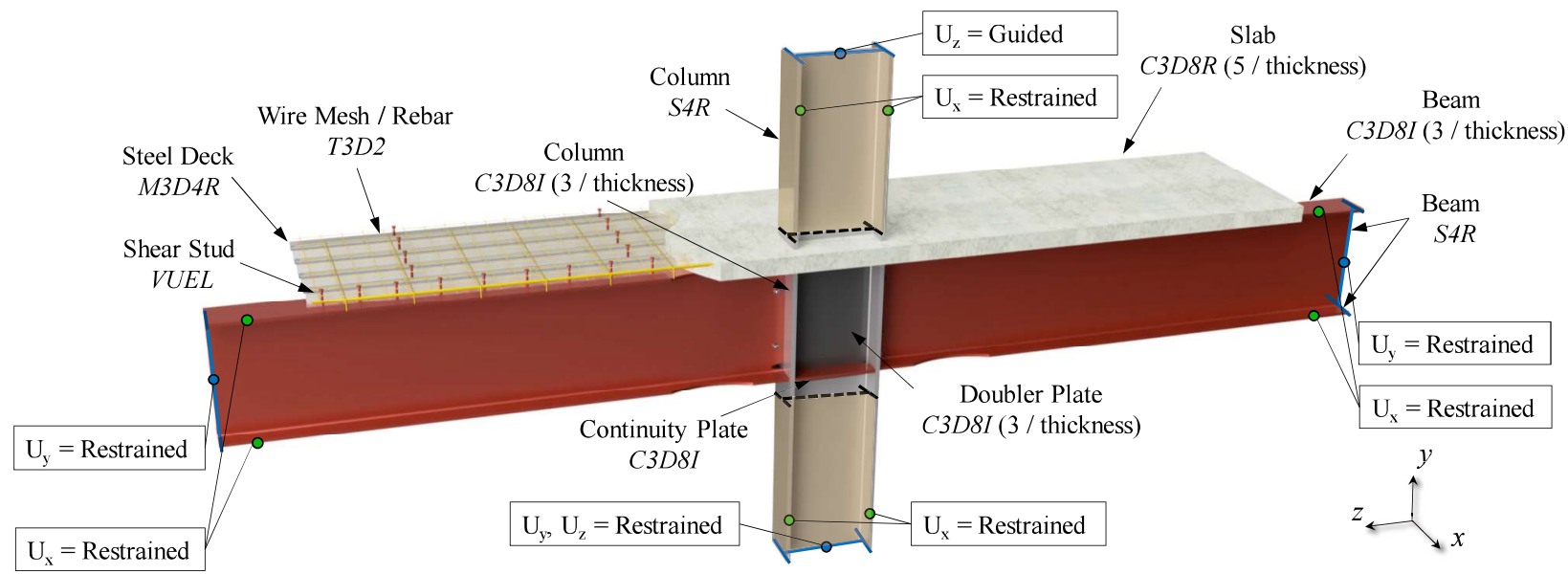

(a)
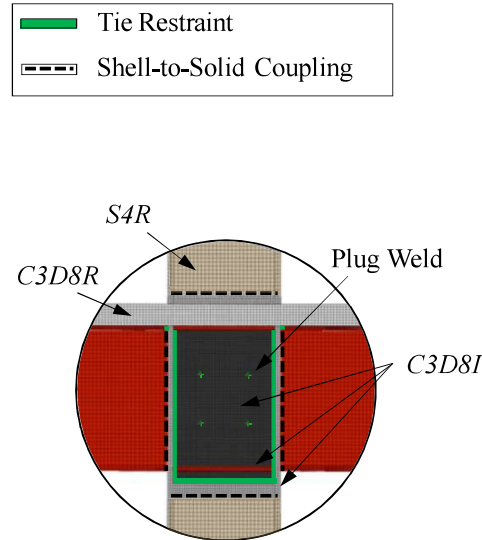

(b)

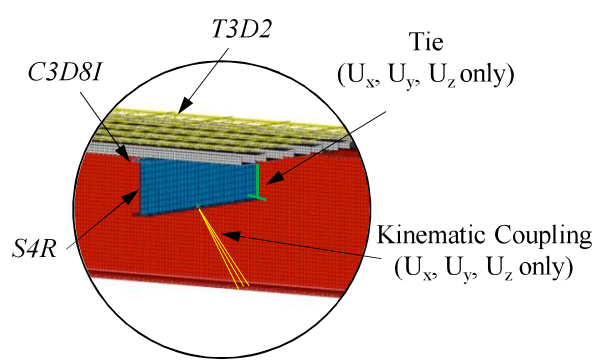

(c)

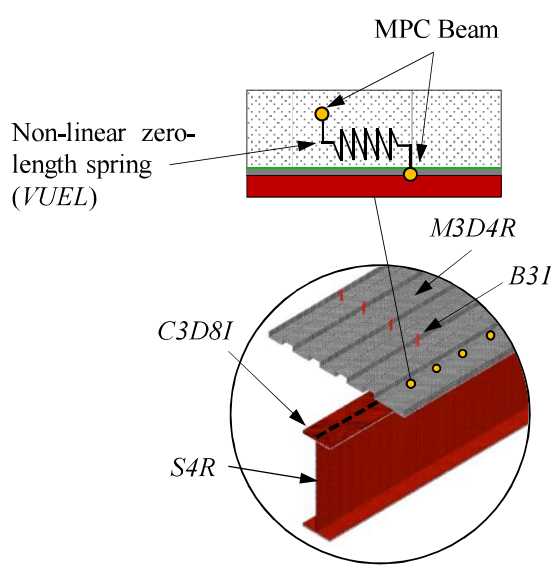

(d)

901 Fig. 1. (a) Continuum finite element model specifics for a typical subassembly configuration with 902 composite floor slab (b) column web panel detail; (c) transverse beams detail; (d) shear studs detail 


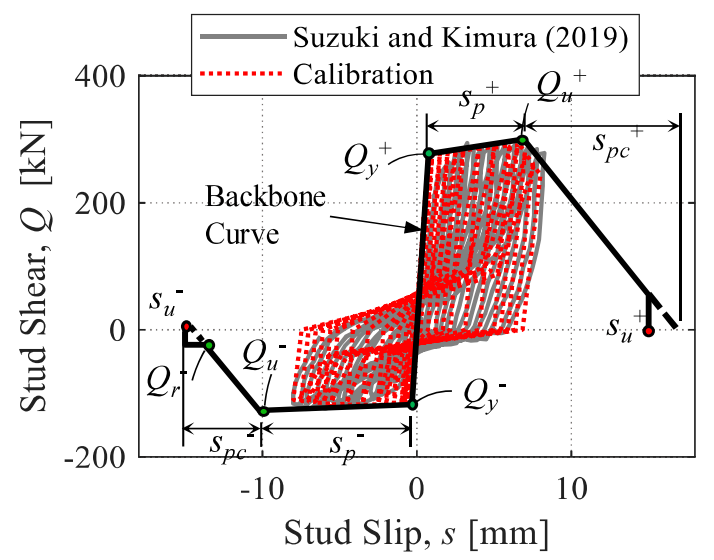

903 Fig. 2. Calibration example of a cluster of four $19 \mathrm{~mm}$ cyclically-loaded shear studs (data values were 904 reproduced from Suzuki and Kimura 2019) 


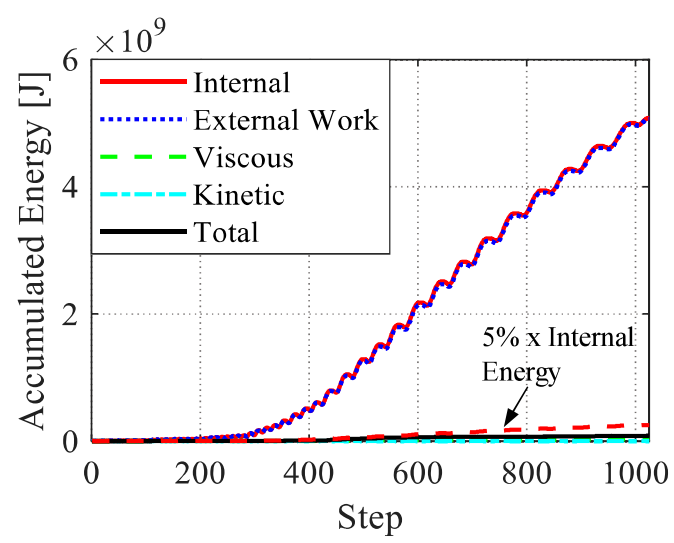

905 Fig. 3. Model energies accumulated throughout the explicit continuum finite element analysis 


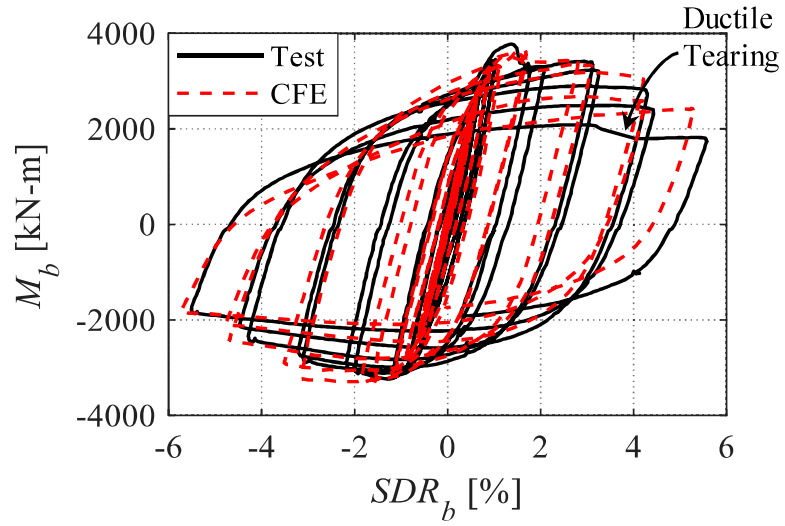

(a)

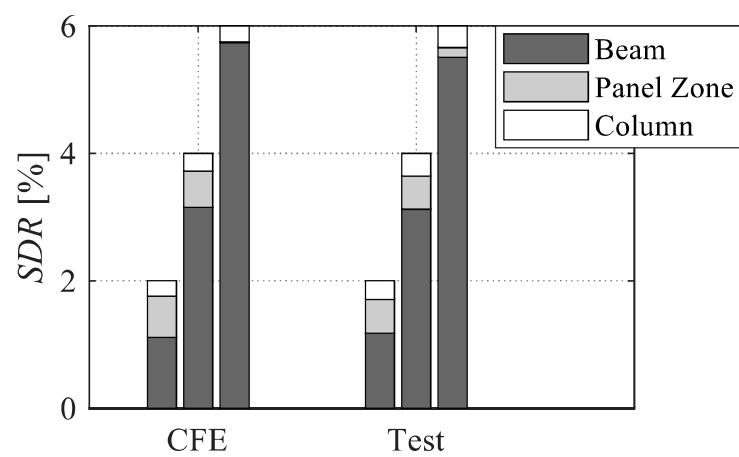

(c)

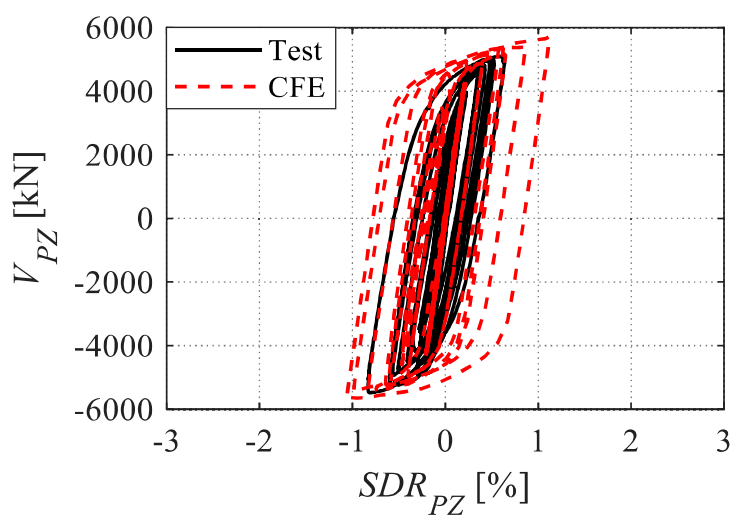

(b)

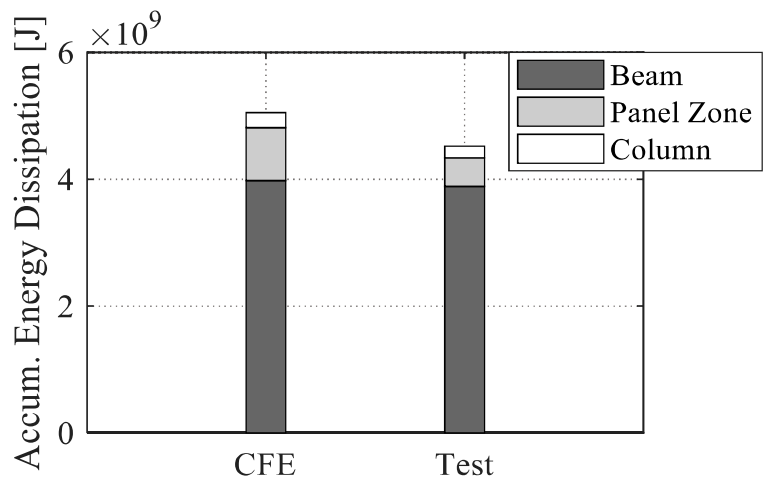

(d)

906 Fig. 4. Comparison between simulated and experimental results (experimental data are reproduced from 907 Zhang et al. 2004) (a) beam hysteretic behavior; (b) panel zone hysteretic behavior; (c) component 908 contribution to the story drift at the first cycle of $2 \%, 4 \%$ and $6 \%$ amplitudes; (d) accumulative energy 909 dissipation in each component 


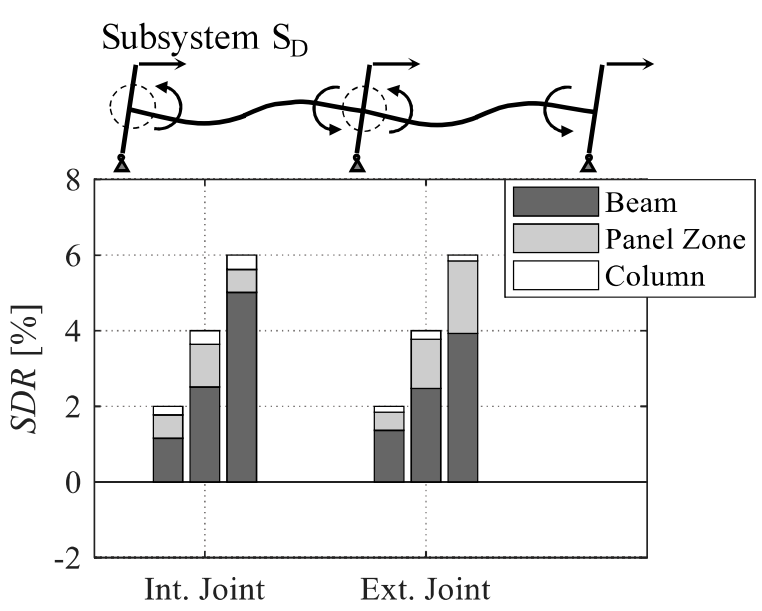

(a)

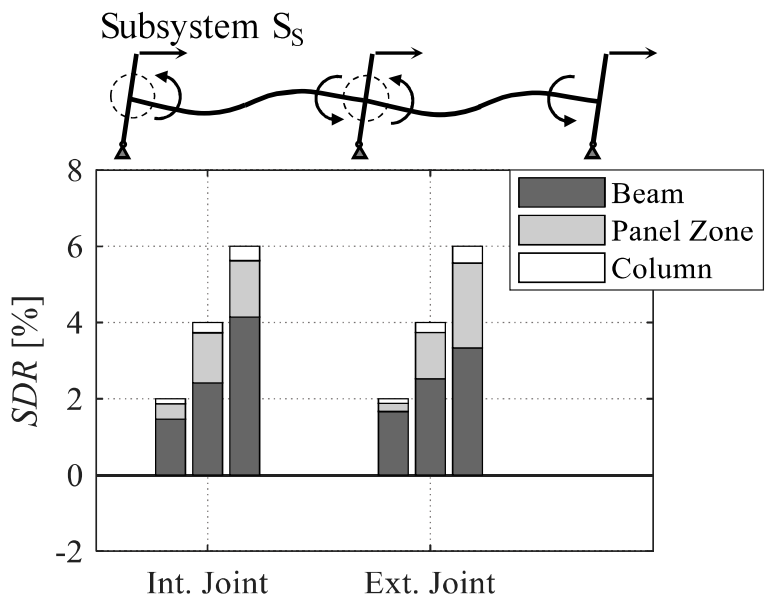

(c)

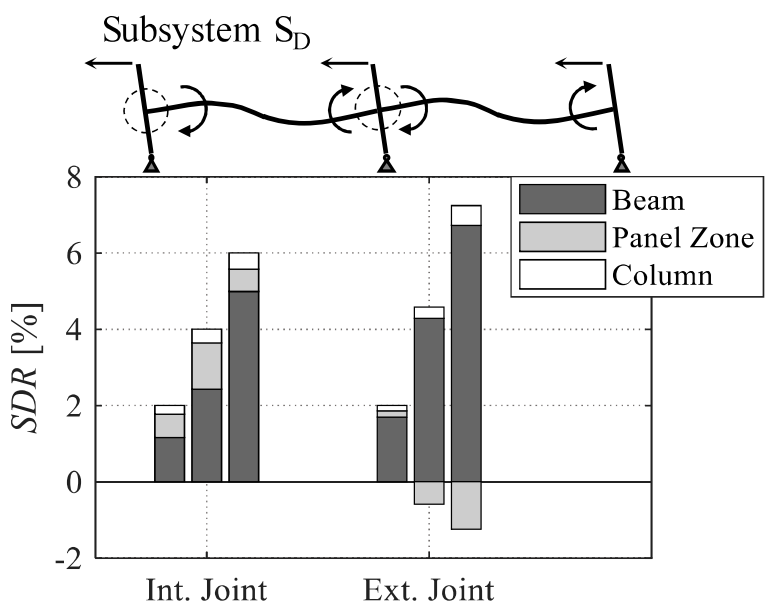

(b)

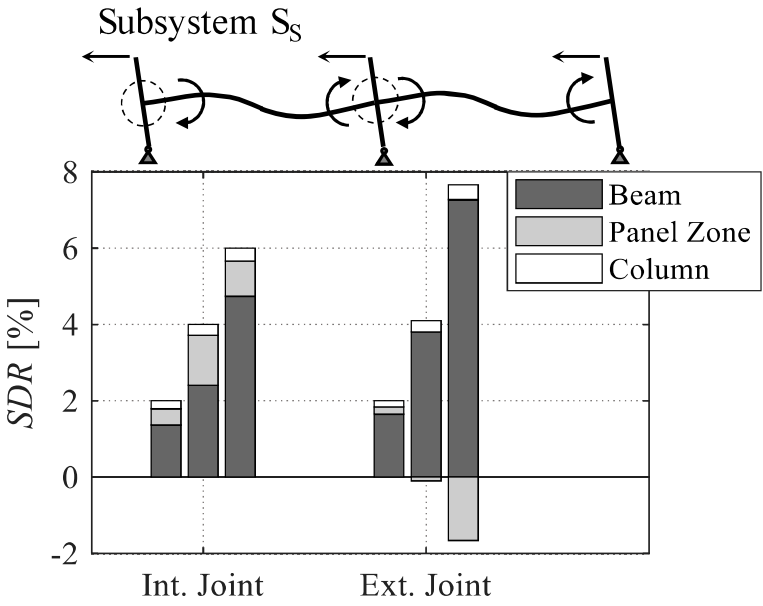

(d)

910 Fig. 5. Component contribution to the story drift at the $2 \%, 4 \%$ and $6 \%$ cycles; (a) subsystem $S_{D}$

911 loading in the east direction; (b) subsystem $S_{D}$ loading in the west direction; (c) subsystem $S_{S}$ loading

912 in the east direction; (d) subsystem $S_{S}$ loading in the west direction 


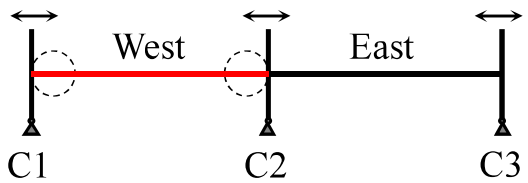

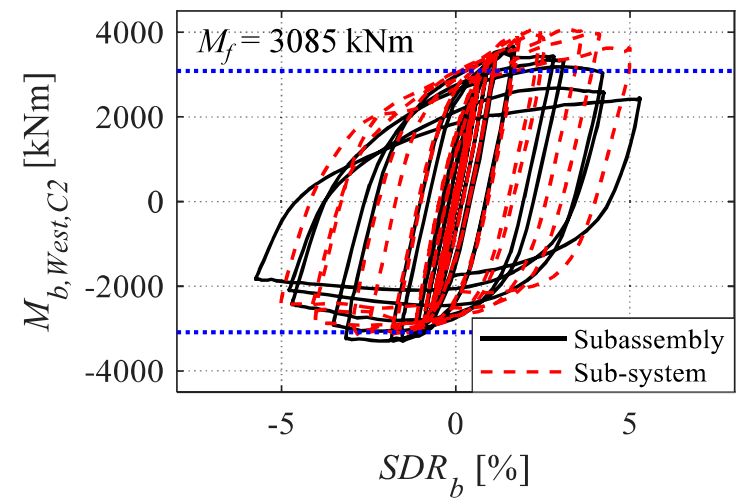

(a) $\mathrm{W} 36 \mathrm{x} 150, \mathrm{~S}_{\mathrm{D}}$ Interior Joint

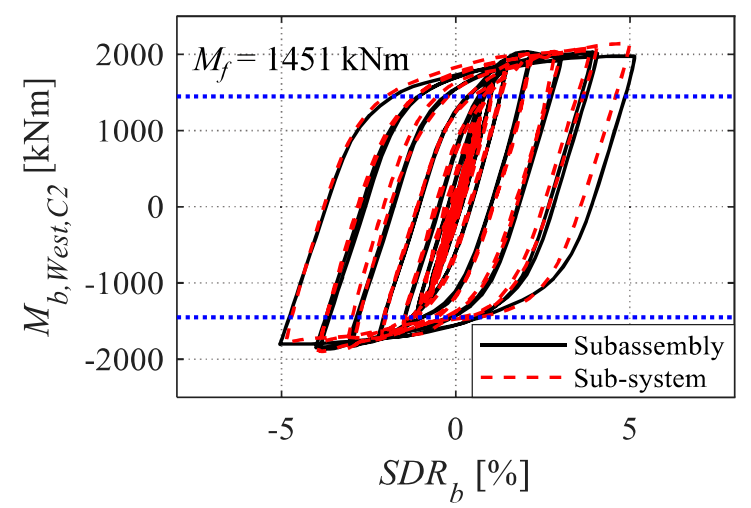

(c) W21x122, S Interior Joint

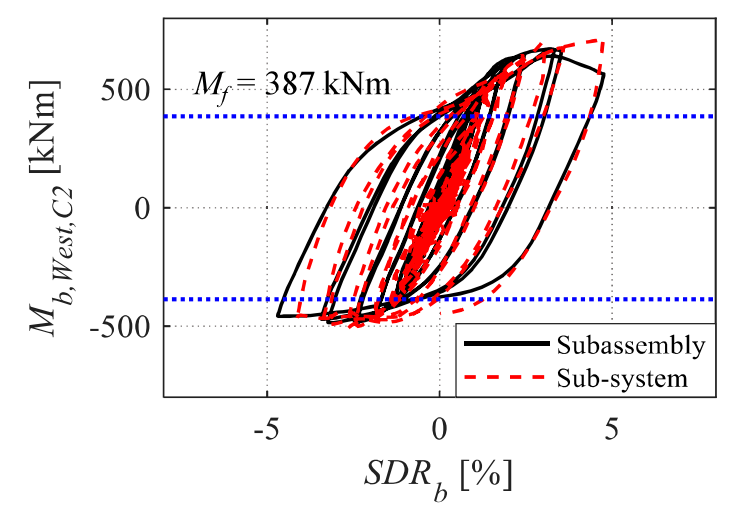

(e) $\mathrm{W} 16 \mathrm{x} 45, \mathrm{~S}_{\mathrm{S}}$ Interior Joint

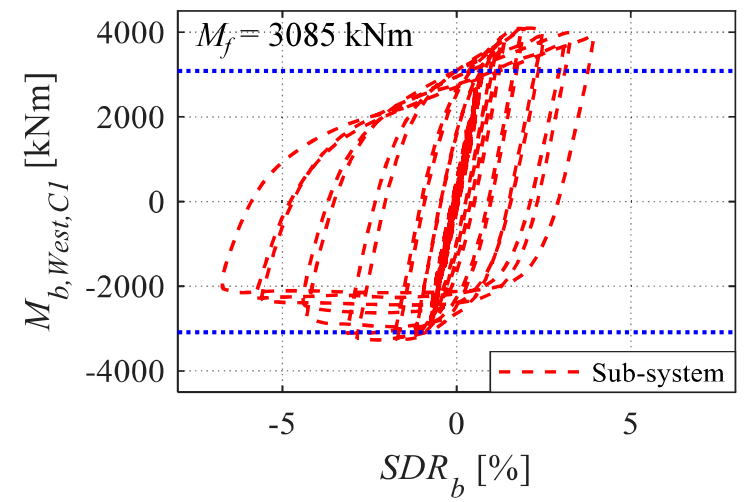

(b) W36x150, $\mathrm{S}_{\mathrm{D}}$ Exterior Joint

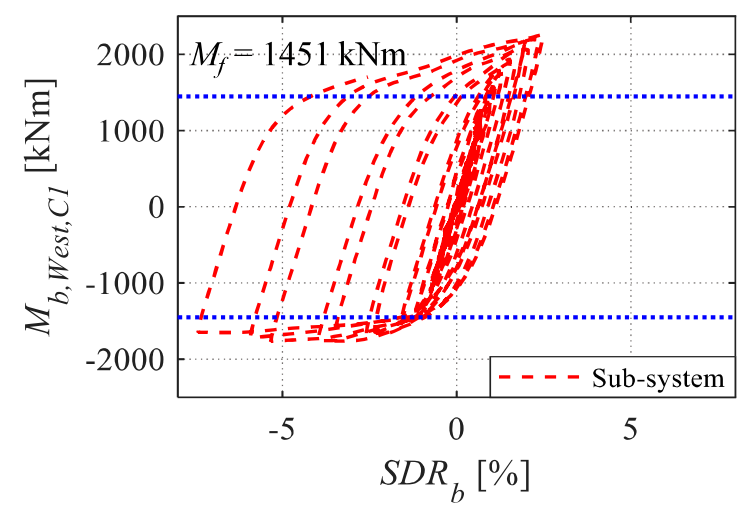

(d) W21x22, $\mathrm{S}_{\mathrm{I}}$ Exterior Joint

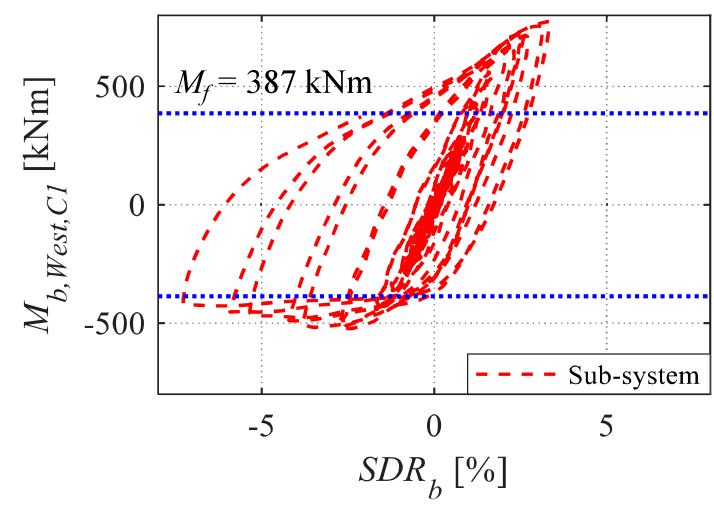

(f) W16x45, $\mathrm{S}_{\mathrm{S}}$ Exterior Joint

913 Fig. 6. West beam hysteretic response - comparisons between sub-system and subassembly response

914 (left: interior joint; right: exterior joint) 


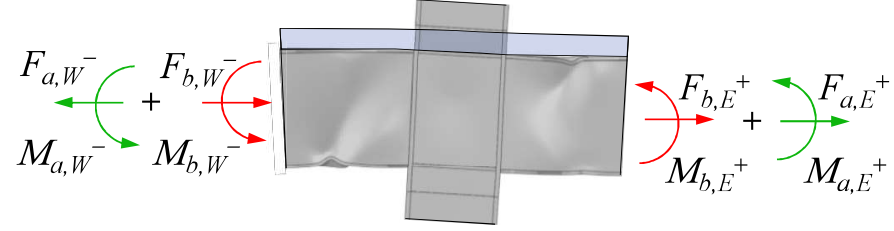

(a) Sub-system, Negative SDR

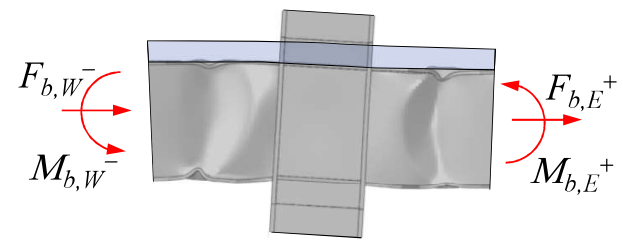

(c) Subassembly, Negative SDR

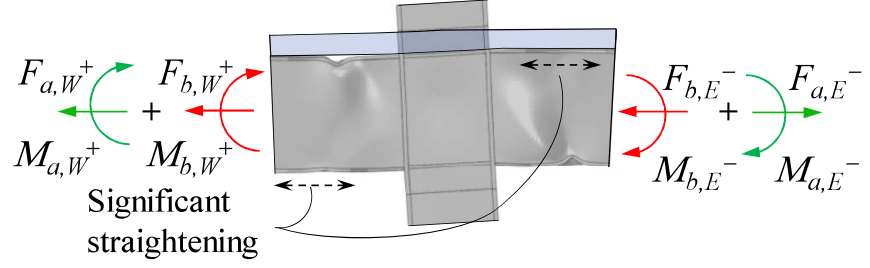

(b) Sub-system, Positive SDR

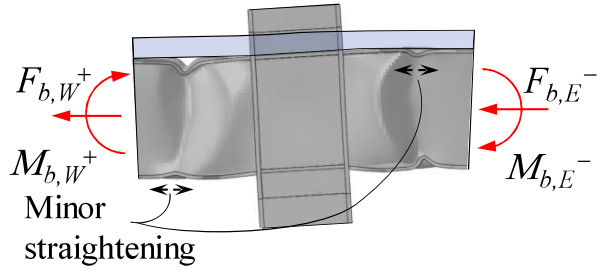

(d) Subassembly, Positive SDR

915 Fig. 7. Straightening mechanism of the buckles in the steel beam upon load reversal 


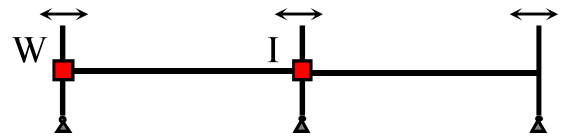

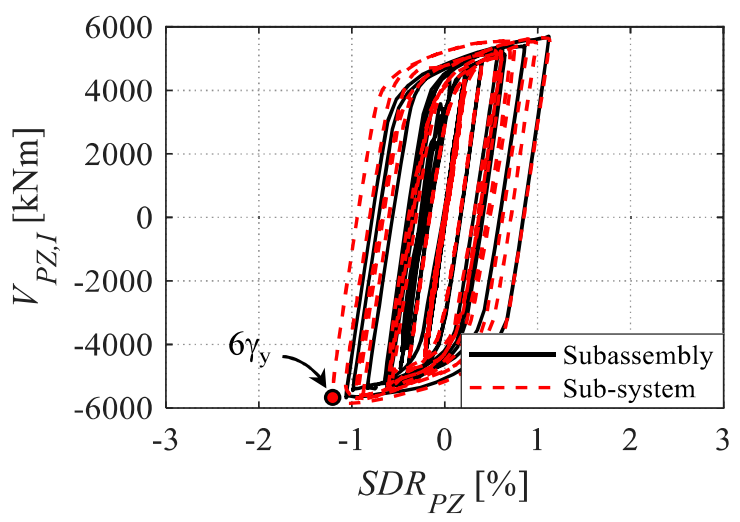

(a) $S_{D}$ Interior Joint

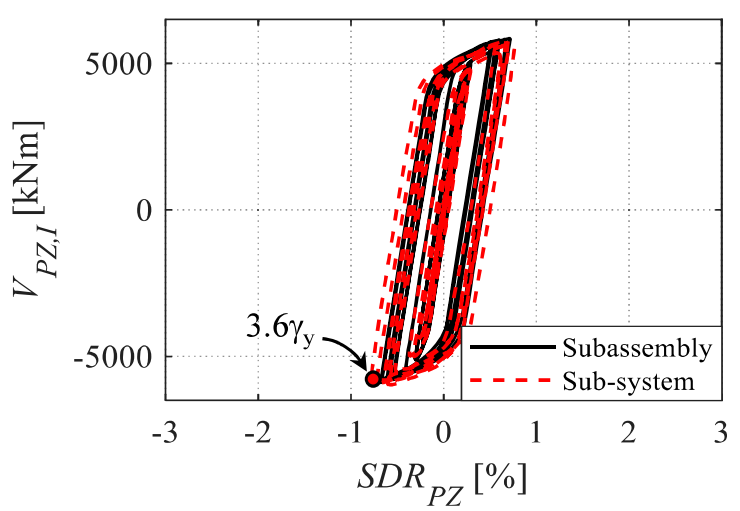

(c) $S_{I}$ Interior Joint

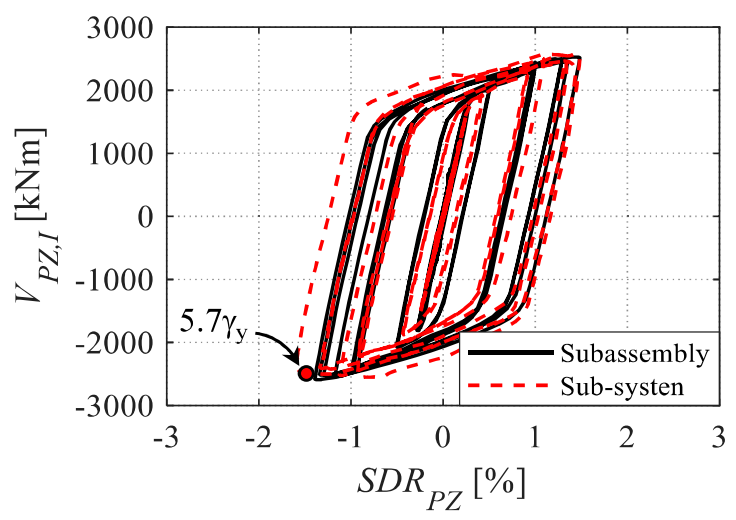

(e) $S_{S}$ Interior Joint

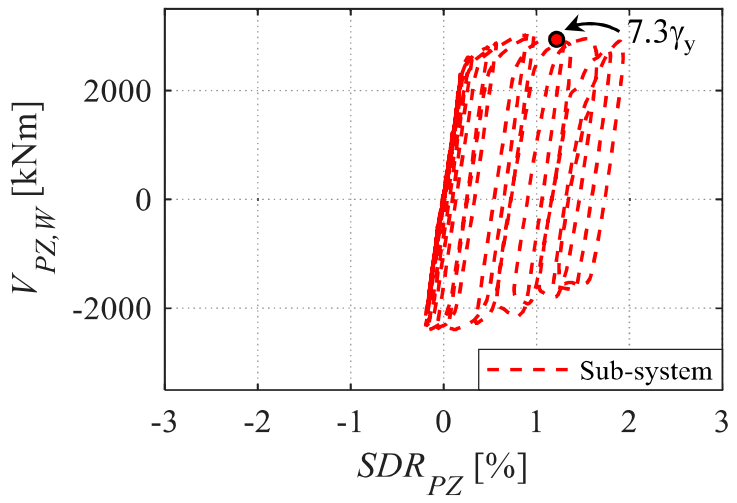

(b) $S_{D}$ Exterior Joint

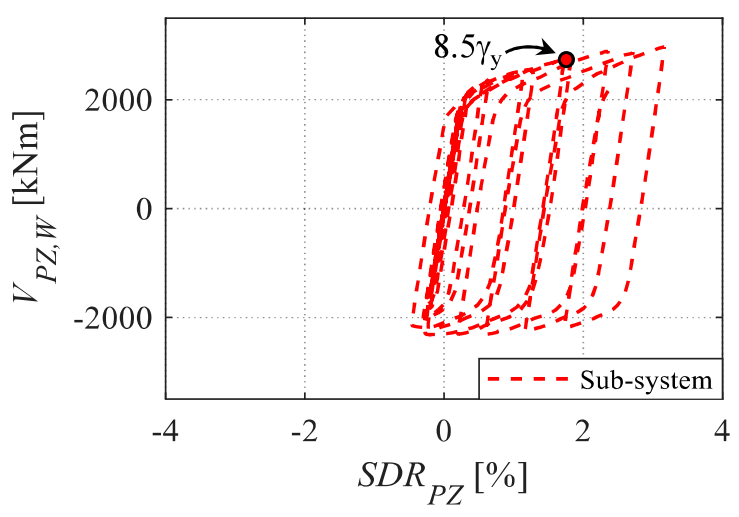

(d) $\mathrm{S}_{\mathrm{I}}$ Exterior Joint

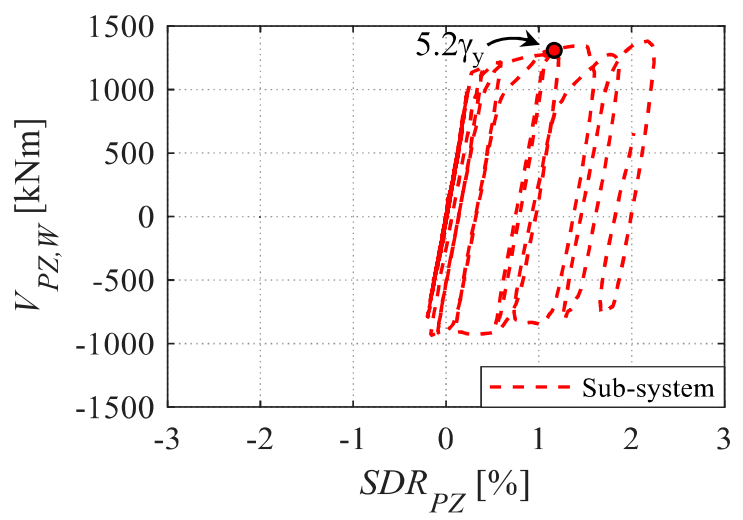

(f) $\mathrm{S}_{\mathrm{S}}$ Exterior Joint

916 Fig. 8. Panel zone hysteretic response - comparisons between sub-system and subassembly response 917 (left: interior joint; right: exterior joint) 


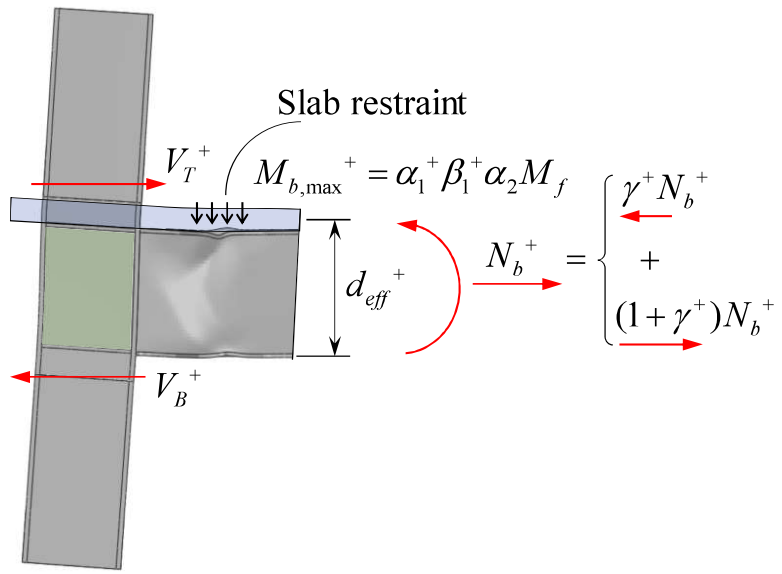

(a) Sagging Excursion

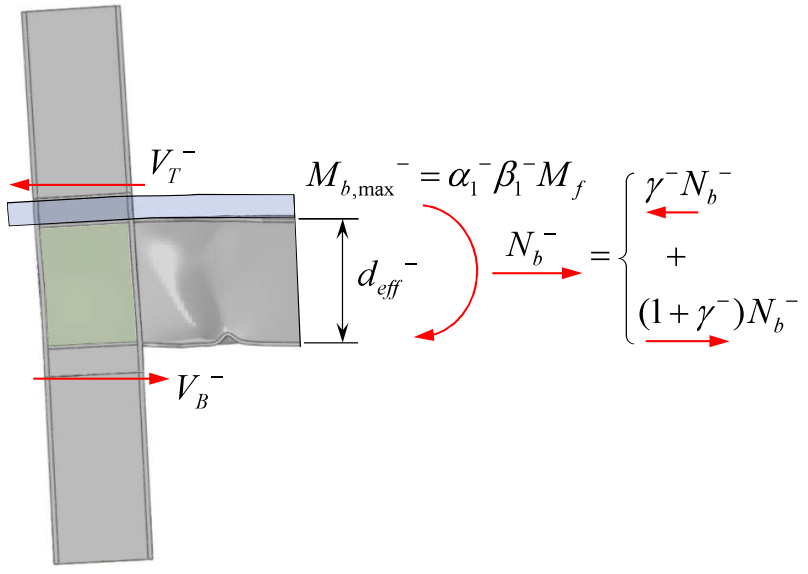

(b) Hogging Excursion

918 Fig. 9. Asymmetric shear demand on the exterior column web panel zone 


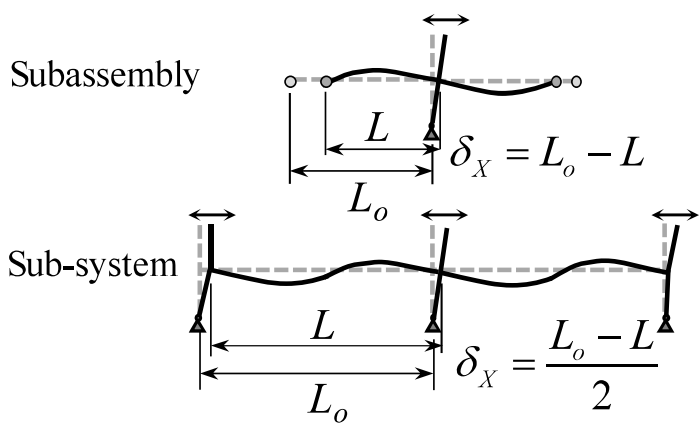

$L$ and $L_{o}$ are measured from the column face at the centerline of the steel beam

(a) Definition

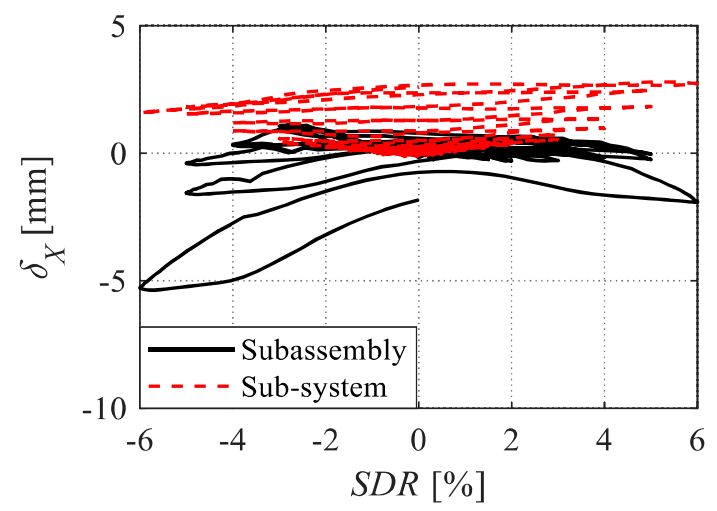

(c) $\mathrm{W} 21 \times 122, \mathrm{~S}_{\mathrm{I}}$

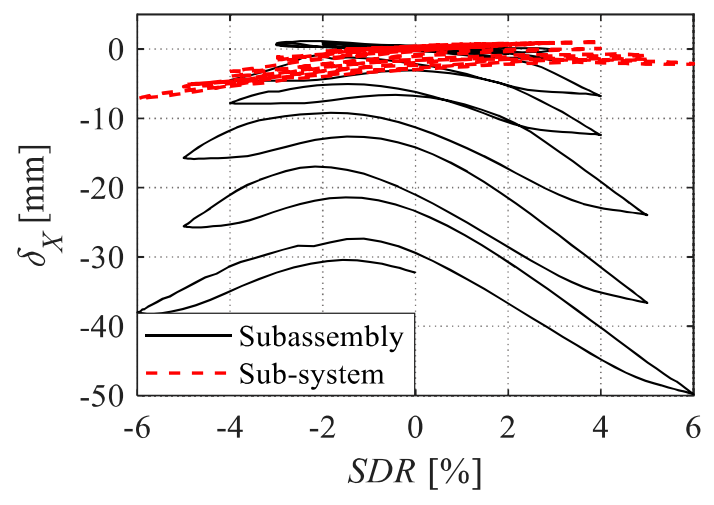

(b) $\mathrm{W} 36 \times 150, \mathrm{~S}_{\mathrm{D}}$

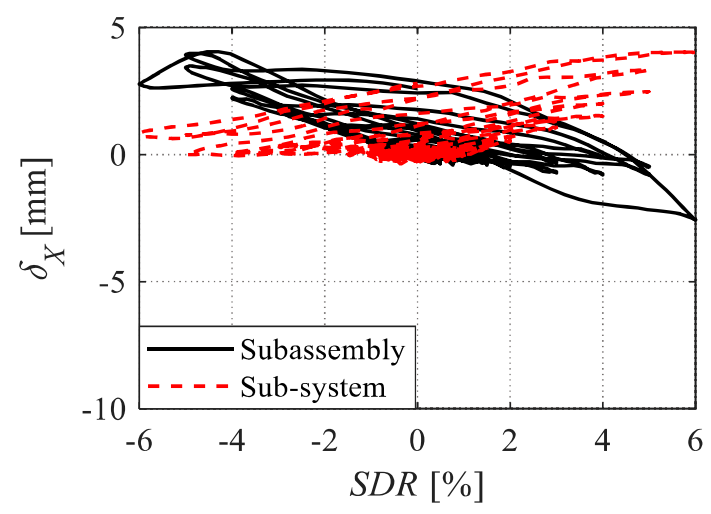

(d) $\mathrm{W} 16 \mathrm{x} 45, \mathrm{~S}_{\mathrm{S}}$

919 Fig. 10. West beam axial shortening - comparisons between sub-system and subassembly response 


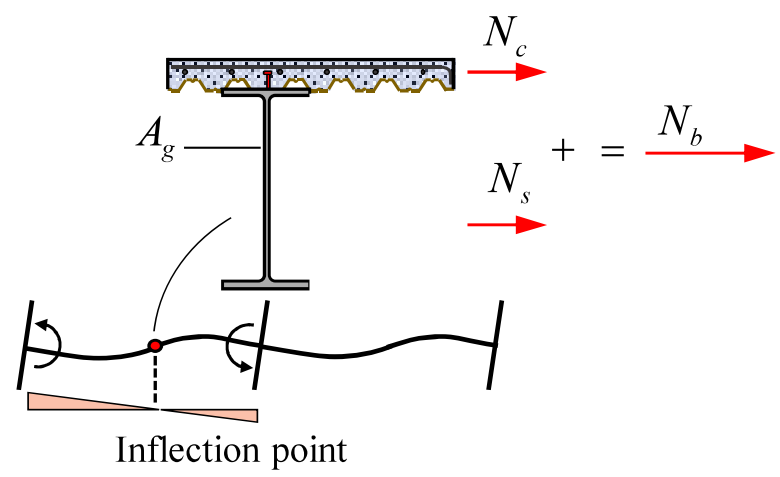

$R F_{y b}$ is the expected yield strength of the steel beam

(a) Definition

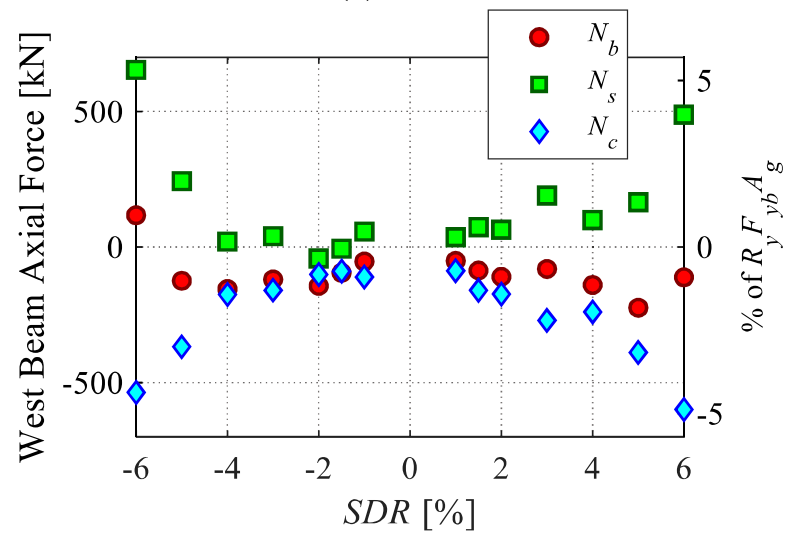

(c) $\mathrm{W} 21 \times 122, \mathrm{~S}_{\mathrm{I}}$

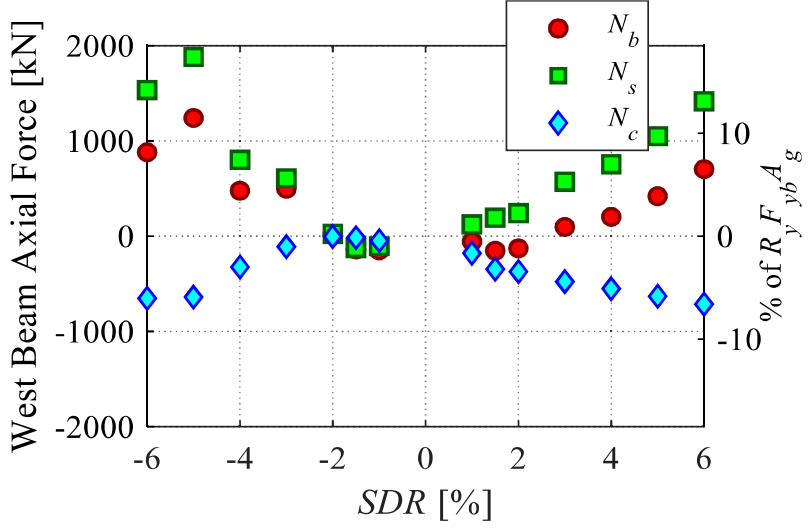

(b) $\mathrm{W} 36 \times 150, \mathrm{~S}_{\mathrm{D}}$

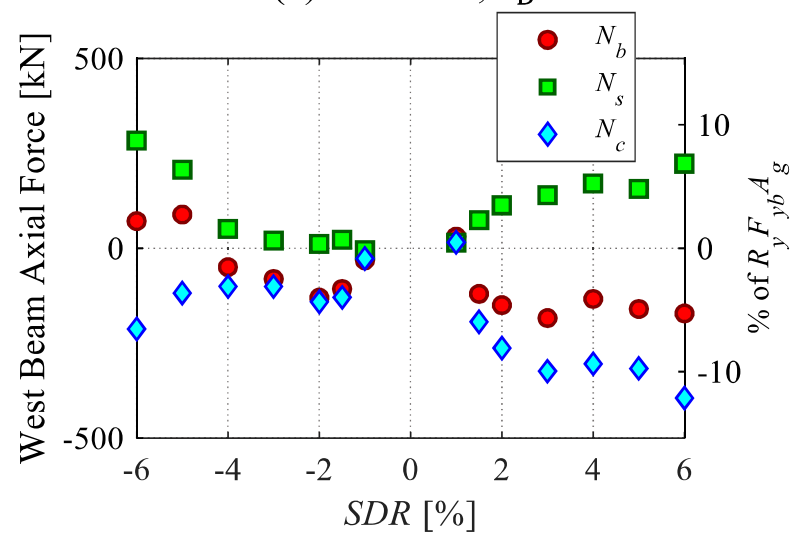

(d) $\mathrm{W} 16 \times 45, \mathrm{~S}_{\mathrm{S}}$

920 Fig. 11. Slab restraint-induced axial forces in the west beam of the sub-systems at peak story drifts 


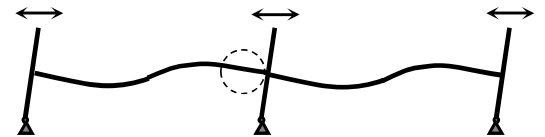

Shear stud closest to column face

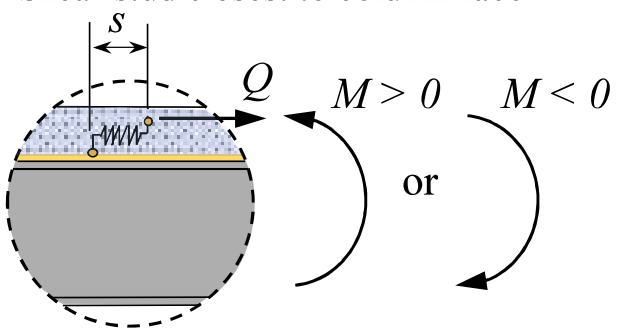

- $\square$ Maximum capacity at $2 \%$ SDR

- o Maximum capacity at 4\% SDR

$\star \star$ Loss of shear capacity

(a) Definition

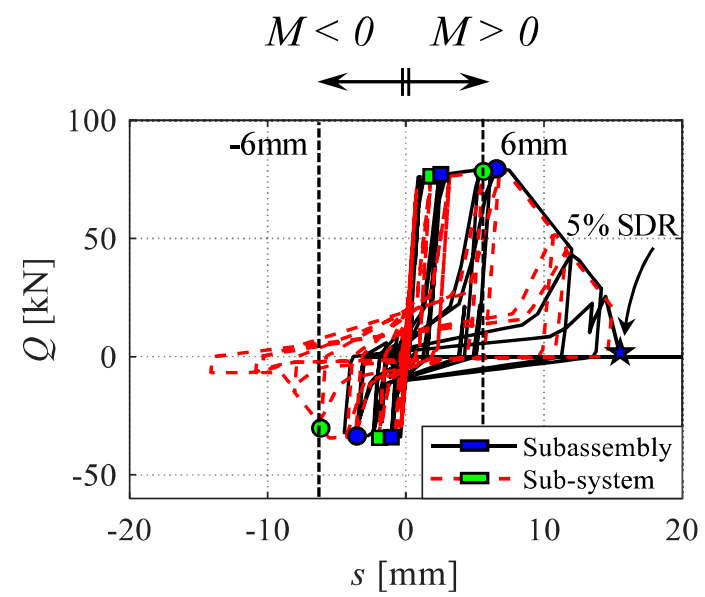

(c) W21x122, $\mathrm{S}_{\mathrm{I}}$

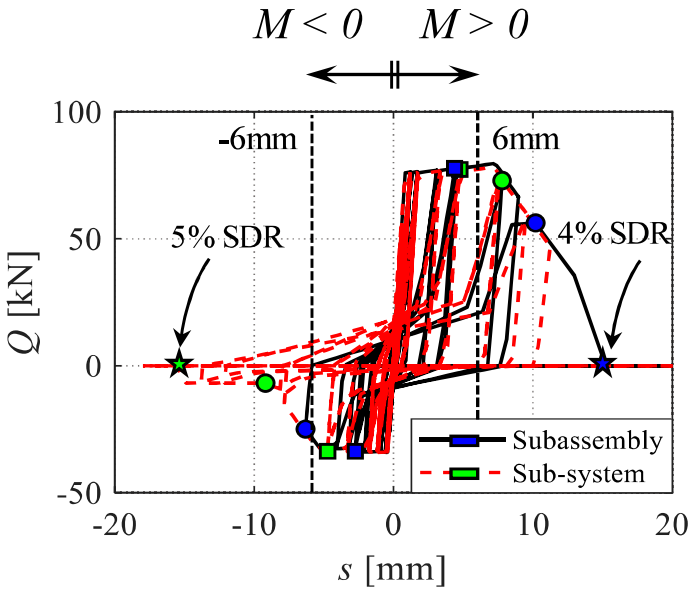

(b) $\mathrm{W} 36 \times 150, \mathrm{~S}_{\mathrm{D}}$

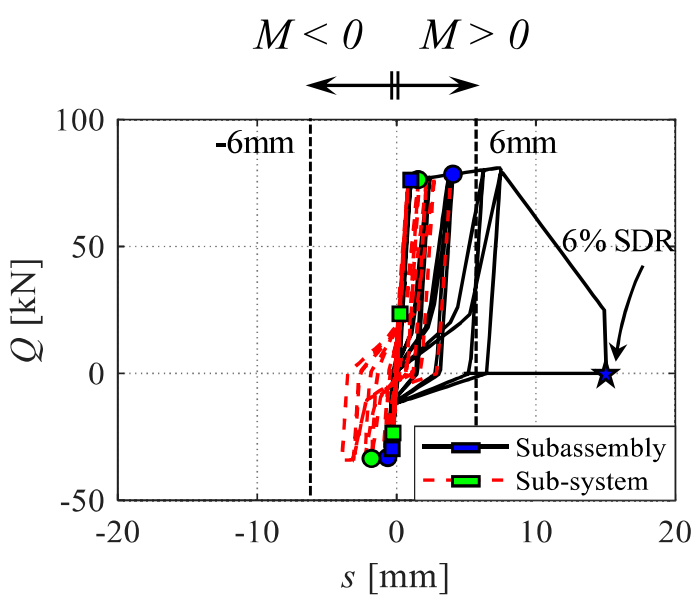

(d) $\mathrm{W} 16 \times 45, \mathrm{~S}_{\mathrm{S}}$

921 Fig. 12. West beam shear stud hysteretic response - comparisons between sub-system and 922 subassembly response 


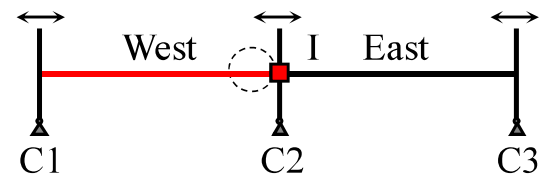

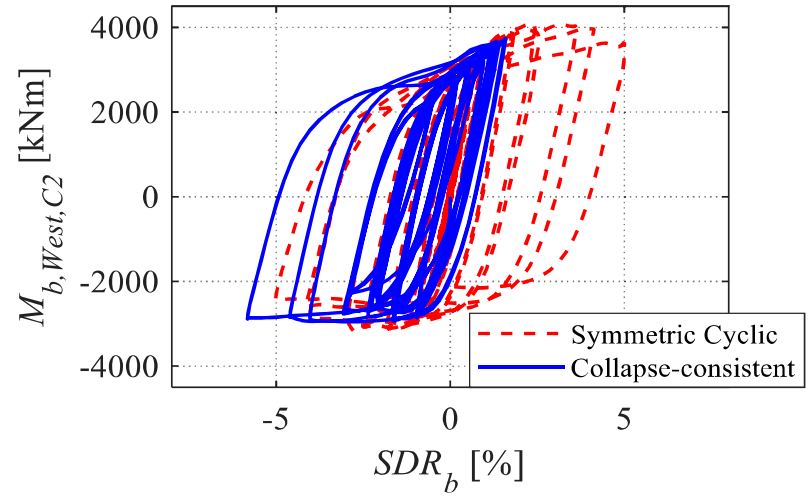

(a) West Beam Hysteretic Response

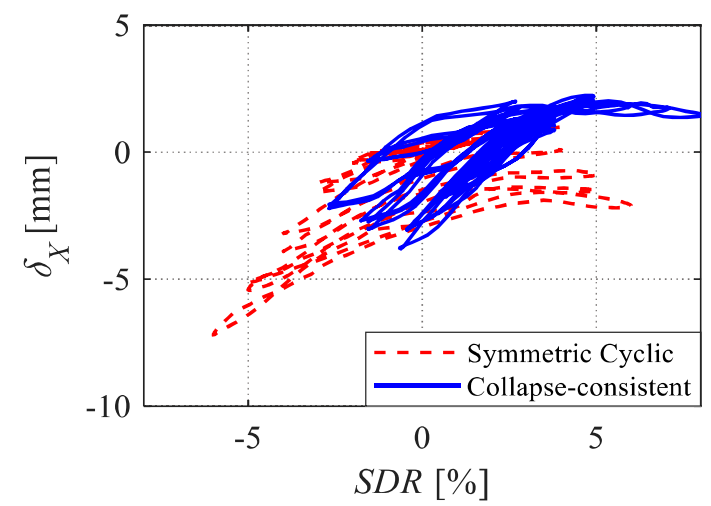

(c) West Beam Axial Shortening

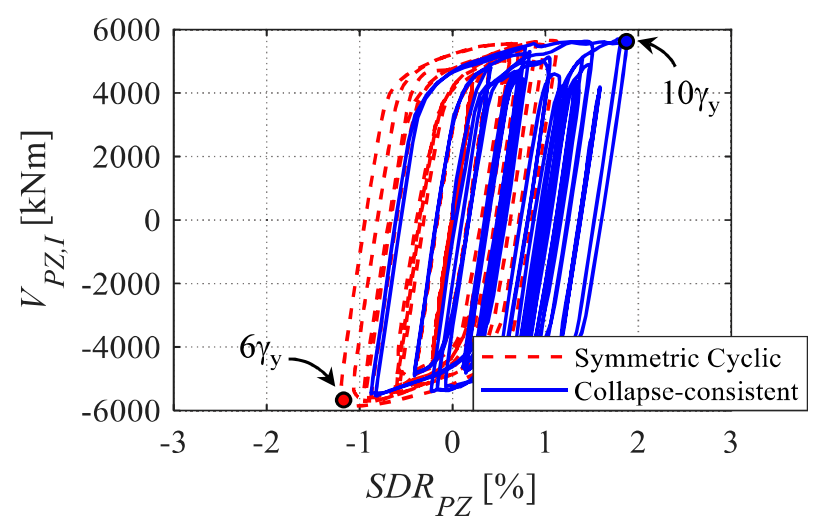

(b) Panel Zone Hysteretic Response

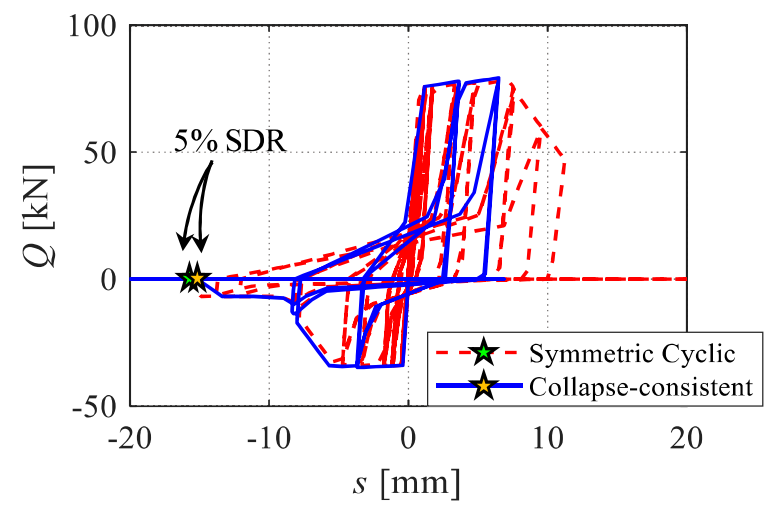

(d) Shear Stud Hysteretic Response

923 Fig. 13. Sub-system $S_{D}$ hysteretic response: comparisons between symmetric cyclic and collapse-

924 consistent loading protocols 